\title{
Spectropolarimetry of Galactic stars with anomalous extinction sightlines ${ }^{\star}$
}

\author{
Aleksandar Cikota ${ }^{1}$, Thiem Hoang ${ }^{2,3}$, Stefan Taubenberger ${ }^{1,4}$, Ferdinando Patat ${ }^{1}$, Paola Mazzei ${ }^{5}$, Nick L.J. Cox ${ }^{6}$, \\ Paula Zelaya ${ }^{7,8}$, Stefan Cikota ${ }^{9,10}$, Lina Tomasella ${ }^{5}$, Stefano Benetti ${ }^{5}$, and Gabriele Rodeghiero ${ }^{11,5}$ \\ ${ }^{1}$ European Southern Observatory, Karl-Schwarzschild-Str. 2, 85748 Garching b. München, Germany \\ e-mail: acikota@eso.org \\ ${ }^{2}$ Korea Astronomy and Space Science Institute, Daejeon 34055, Korea \\ e-mail: thiemhoang@kasi.re.kr \\ ${ }^{3}$ Korea University of Science and Technology, 217 Gajungro, Yuseong-gu, Daejeon 34113, Korea \\ ${ }^{4}$ Max-Planck-Institut für Astrophysik, Karl-Schwarzschild-Str. 1, 85741 Garching b. München, Germany \\ 5 INAF-Osservatorio Astronomico di Padova, Vicolo dell'Osservatorio 5, 35122 Padova, Italy \\ ${ }^{6}$ Anton Pannekoek Institute for Astronomy, University of Amsterdam, 1090 GE Amsterdam, The Netherlands \\ ${ }^{7}$ MAS-Millennium Institute of Astrophysics, Casilla 36-D, 7591245 Santiago, Chile \\ ${ }^{8}$ Instituto de Astrofísica, Pontificia Universidad Católica de Chile, Casilla 306, Santiago 22, Chile \\ ${ }^{9}$ University of Zagreb, Faculty of Electrical Engineering and Computing, Department of Applied Physics, Unska 3, \\ 10000 Zagreb, Croatia \\ ${ }^{10}$ Ruđer Bošković Institute, Bijenička cesta 54, 10000 Zagreb, Croatia \\ ${ }^{11}$ Max-Planck-Institut für Astronomie, Königstuhl 17, 69117 Heidelberg, Germany
}

Received 18 June 2017 / Accepted 20 march 2018

\begin{abstract}
Highly reddened type Ia supernovae (SNe Ia) with low total-to-selective visual extinction ratio values, $R_{V}$, also show peculiar linear polarization wavelength dependencies with peak polarizations at short wavelengths $\left(\lambda_{\max } \lesssim 0.4 \mu \mathrm{m}\right)$. It is not clear why sightlines to SNe Ia display such different continuum polarization profiles from interstellar sightlines in the Milky Way with similar $R_{V}$ values. We investigate polarization profiles of a sample of Galactic stars with low $R_{V}$ values, along anomalous extinction sightlines, with the aim to find similarities to the polarization profiles that we observe in SN Ia sightlines. We undertook spectropolarimetry of 14 stars, used archival data for 3 additional stars, and ran dust extinction and polarization simulations (by adopting the picket-fence alignment model) to infer a simple dust model (size distribution, alignment) that can reproduce the observed extinction and polarization curves. Our sample of Galactic stars with low $R_{V}$ values and anomalous extinction sightlines displays normal polarization profiles with an average $\lambda_{\max } \sim 0.53 \mu \mathrm{m}$, and is consistent within $3 \sigma$ to a larger coherent sample of Galactic stars from the literature. Despite the low $R_{V}$ values of dust toward the stars in our sample, the polarization curves do not show any similarity to the continuum polarization curves observed toward SNe Ia with low $R_{V}$ values. There is a correlation between the best-fit Serkowski parameters $K$ and $\lambda_{\max }$, but we did not find any significant correlation between $R_{V}$ and $\lambda_{\max }$. Our simulations show that the $K-\lambda_{\max }$ relationship is an intrinsic property of polarization. Furthermore, we have shown that in order to reproduce polarization curves with normal $\lambda_{\max }$ and low $R_{V}$ values, a population of large $(\mathrm{a} \geq 0.1 \mu \mathrm{m})$ interstellar silicate grains must be contained in the dust composition.
\end{abstract}

Key words. polarization - ISM: general - dust, extinction - supernovae: general - galaxies: ISM

\section{Introduction}

The motivation to study anomalous sightlines toward highly reddened Galactic stars derives from type Ia supernova (SN) observations that show peculiar extinction curves with very low $R_{V}$ values, as well as peculiar polarization wavelength dependencies (polarization curves).

Past studies that included large samples of SNe Ia showed that the total-to-selective visual extinction ratio, $R_{V}$, of dust in type Ia $\mathrm{SN}$ host galaxies ranges from 1 to 3.5 and is in most cases lower than the average value of Milky Way dust, $R_{V} \sim 3.1$ (Riess et al. 1996; Phillips et al. 1999; Altavilla et al. 2004; Reindl et al. 2005; Conley et al. 2007; Wang et al. 2006; Goobar 2008; Nobili \& Goobar 2008; Kessler et al. 2009;

* The reduced spectra are only available at the CDS via anonymous ftp to cdsarc.u-strasbg.fr (130.79.128.5) or via http://cdsarc.u-strasbg.fr/viz-bin/qcat?J/A+A/615/A42
Hicken et al. 2009; Folatelli et al. 2010; Lampeitl et al. 2010; Mandel et al. 2011; Cikota et al. 2016).

Observations of individual highly reddened $\mathrm{SNe}$ Ia also reveal host galaxy dust with low $R_{V}$ values, for instance, $R_{V}=2.57_{-0.21}^{+0.23}$ for the line of sight of SN 1986G (Phillips et al. 2013), $R_{V} \sim 1.48$ for SN 2006X (Wang et al. 2008), $R_{V}=1.20{ }_{-0.14}^{+0.26}$ for SN 2008fp (Phillips et al. 2013), and $R_{V}^{o b s}=1.64 \pm 0.16$ for SN 2014J (Foley et al. 2014).

Linear (spectro)polarimetric observations of these four $\mathrm{SNe}$ also display anomalous interstellar polarization curves (Patat et al. 2015), steeply rising toward blue wavelengths. Patat et al. (2015) fitted their observations with a Serkowski curve (Serkowski et al. 1975) to characterize the polarization curves.

The Serkowski curve is an empirical wavelength dependence of interstellar linear polarization:

$$
\frac{P(\lambda)}{P_{\max }}=\exp \left[-K \ln ^{2}\left(\frac{\lambda_{\max }}{\lambda}\right)\right]
$$


The wavelength of peak polarization, $\lambda_{\max }$, depends on the dust grain size distribution. For an enhanced abundance of small dust grains, $\lambda_{\max }$ moves to shorter wavelengths, and for an enhanced abundance of large dust grains, it moves to longer wavelengths. Thus, linear spectropolarimetry probes the alignment of dust grains and the size distribution of the aligned dust grains.

Patat et al. (2015) found that the polarization curve of all $4 \mathrm{SNe}$ display an anomalous behavior, with $\lambda_{\max } \sim 0.43 \mu \mathrm{m}$ for SN 1986G, and $\lambda_{\max } \lesssim 0.4 \mu \mathrm{m}$ for SN 2006X, SN 2008fp, and SN 2014J. Because SNe Ia have a negligible intrinsic continuum polarization (Wang \& Wheeler 2008), the anomalous polarization curves likely have to be associated with the properties of host galaxies dust. Zelaya et al. (2017) expanded the sample of 4 SNe Ia investigated in Patat et al. (2015), and presented a study of 19 type Ia SNe. They grouped the SNe into the "sodium-sample", consisting of $12 \mathrm{SNe}$ that show higher continuum polarization values and interstellar $\mathrm{Na} \mathrm{I} \mathrm{D} \mathrm{lines} \mathrm{at}$ the redshift of their host galaxies, and the "non-sodium-sample" with no rest-frame $\mathrm{Na} I \mathrm{D}$ lines and smaller peak polarization. Eight sodium-sample SNe have $\lambda_{\max } \lesssim 0.4 \mu \mathrm{m}$, and their polarization angles are aligned with the spiral arms of their host galaxies, which is evidence that the polarizing dust is likely located in their host galaxies and aligned due to the magnetic fields of the host galaxies. The non-sodium-sample $\mathrm{SNe}$ are less strongly polarized, with $P_{\max } \lesssim 0.5 \%$, have $\lambda_{\max }$ values similar to the common Galactic dust (with $\lambda_{\max } \sim 0.55 \mu \mathrm{m}$ ), and their polarization angles do not align with host-galaxy features, which might be interpreted as the continuum polarization being produced by the Galactic foreground dust.

It is not understood why these reddened SN Ia sightlines show such a different polarization profile compared to the typical Milky Way dust. A natural explanation is that the composition of dust in the SN Ia host galaxies is different from that in the Galaxy.

However, there are alternative explanations. Scattering might explain the low $R_{V}$ values as well as the peculiar polarization profiles. As illustrated by Patat et al. (2015) (see their Fig. 6), the polarization profile of SN 2006X may, in addition to the Serkowski component, also have a component induced by Rayleigh scattering. However, if a light echo propagates through local dust, we expect to observe variability in $R_{V}$ and polarization (Wang 2005), which is usually not the case (see Fig. 4 in Zelaya et al. 2017). Yang et al. (2017) used observations made with the Hubble Space Telescope (HST) to map the interstellar medium (ISM) around SN 2014J through light echoes. These authors observed two echo components: a diffuse ring and a luminous arc, produced through dust scattering of different grain sizes. From the wavelength dependence of the scattering optical depth, the arc dust favors a low $R_{V}$ value of $\sim 1.4$, which is consistent with the $R_{V}$ measured along the direct line of sight, while the ring is consistent with a common Milky Way $R_{V} \sim 3$ value.

Another interesting explanation for the peculiar $\mathrm{SNe}$ Ia sightlines is given by Hoang (2017), who simultaneously fit a two-component (interstellar and circumstellar) extinction and polarization model to photometric and (spectro-) polarimetric observations of SNe 1986G, 2006X, 2008fp, and 2014J to investigate the grain size distribution and alignment functions of dust along these lines of sights. Hoang (2017) were able to reproduce the observational data of SN 1986G and SN 2006X by assuming an enhanced abundance of small silicate grains in the interstellar dust only, while in the case of SN 2014J, a contribution of circumstellar (CS) dust must be accounted for.
In the case of SN 2008fp, Hoang (2017) found that the alignment of small dust grains must be as efficient as that of large grains, but the existence of CS dust is uncertain. Hoang (2017) suggested that the enhanced abundance of small silicate grains might be produced by cloud collisions driven by the $\mathrm{SN}$ radiation pressure. Strong SN radiation might also induce efficient alignment of small grains via the radiative torque mechanism. However, in the case of alignment via the radiative torque mechanism, the polarization angle alignment with host-galaxy features remains unexplained.

The aim of this work is to investigate Galactic stars with low $R_{V}$ values with spectropolarimetry, in order to possibly find similarities to the polarization curves observed toward SNe Ia. Numerical simulations are used to infer general properties of interstellar dust toward these stars by simultaneously fitting to extinction curves with low $R_{V}$ values and normal polarization curves.

The paper is structured as follows: in Sect. 2 we describe our sample of stars, in Sect. 3 the instruments and observing strategies, in Sect. 4 we present the data processing and results, in Sect. 5 the analysis of the observations, in Sect. 6 we run simulations in order to interpret the observed data, in Sect. 7 we discuss the results, and finally we summarize and conclude in Sect. 8.

\section{Target sample}

We selected our targets from the samples presented by Mazzei \& Barbaro (2008, 2011). Mazzei \& Barbaro (2011) obtained 785 extinction curves for sightlines with $E(B-$ $V) \geqslant 0.2 \mathrm{mag}$ (Savage et al. 1985), observed with the Astronomical Netherlands Satellite (ANS) in five UV bands $\left(1 / \lambda=6.46,5.56,4.55,4.01\right.$ and $3.04 \mu \mathrm{m}^{-1}$; Wesselius et al. 1982). They combined the UV observations with Two-Micron All-Sky Survey (2MASS) observations in the near-infrared $J, H$, and $K$ bands, applied a least-squares fit of the standard Cardelli et al. (1989) extinction curve (CCM) with different $R_{V}$ values, and determined the residual differences between the observed values and best-fit CCM curve at five UV wavelengths. The curves were classified as anomalous if at least one UV wavelength deviated by more than $2 \sigma$ from the best-fit standard CCM curve. Twenty curves with weaker UV bumps and steeper far-UV slopes (type A), or with stronger bumps and smoother far-UV rises (type B) compared to their best-fit CCM curve, were analyzed in Mazzei \& Barbaro (2008). Mazzei \& Barbaro (2011) focus on 64 lines of sight for which the corresponding bestfit CCM curve is always well below $(\geqslant 2 \sigma)$ or well above the observed data (type $C$ curves), with some exception at $1 / \lambda=3.01$ for five curves (see bottom panel of Fig. 1 in Mazzei \& Barbaro 2011). They conclude that the sightlines characterized by anomalous type $C$ extinction curves require lower dust abundances than environments characterized by normal CCM extinction curves.

From these 64 anomalous lines of sight, we selected 14 lines of sight with the lowest $R_{V}$ values and observed them with the FOcal Reducer and low dispersion Spectrograph (FORS2), the Asiago Faint Object Spectrograph and Camera (AFOSC), and the Calar Alto Faint Object Spectrograph (CAFOS). The observed targets are listed in Table 1. Additionally, we used archival HPOL data for 3 stars.

\section{Instruments and methods}

We observed our targets using three different instruments and telescopes: the FOcal Reducer and low dispersion Spectrograph 
Table 1. Observed stars.

\begin{tabular}{|c|c|c|c|c|c|c|c|c|c|}
\hline Name & $\begin{array}{l}\text { RA } \\
\text { (J2000) }\end{array}$ & $\begin{array}{l}\text { Dec } \\
(\mathrm{J} 2000)\end{array}$ & $\begin{array}{r}\mathrm{V} \\
(\mathrm{mag})\end{array}$ & $\begin{array}{l}\text { B-V } \\
(\mathrm{mag})\end{array}$ & $\begin{array}{l}\text { Spec. } \\
\text { type }\end{array}$ & Type & Telescope & $\begin{array}{l}\text { No. of } \\
\text { Epochs }\end{array}$ & Comment \\
\hline $\mathrm{BD}+233762$ & 194542.31 & +235904.0 & 9.34 & 0.62 & B0.5III & SCI & CAHA & 1 & HD 344880 , star in association \\
\hline $\mathrm{BD}+453341$ & 205702.68 & +463244.7 & 8.73 & 0.38 & B1II & SCI & CAHA & 2 & \\
\hline HD 1337 & 001743.06 & +512559.1 & 6.14 & -0.13 & $\mathrm{O} 9.2 \mathrm{II}+\mathrm{O} 8 \mathrm{~V}$ & SCI & $\begin{array}{l}\text { CAHA } \\
\text { Asiago }\end{array}$ & $\begin{array}{l}3 \\
2\end{array}$ & W UMa type \\
\hline HD 137569 & 152620.82 & +144136.3 & 7.91 & -0.05 & B9Iab:p & SCI & $\begin{array}{l}\text { CAHA } \\
\text { Asiago } \\
\text { VLT }\end{array}$ & $\begin{array}{l}2 \\
2 \\
2 \text { free }+4 \text { GG435 }\end{array}$ & Post-AGB star (proto-PN) \\
\hline HD 144579 & 160456.79 & +390923.4 & 6.67 & 0.73 & G8V & unPolStd & $\begin{array}{l}\text { CAHA } \\
\text { Asiago }\end{array}$ & $\begin{array}{l}2 \\
1\end{array}$ & High proper motion \\
\hline HD 154445 & 170532.26 & -005331.5 & 5.61 & 0.12 & B1V & PolStd & CAHA & 2 & HR 6353 \\
\hline HD 194092 & 202205.44 & +405908.2 & 8.28 & 0.09 & B0.5III & SCI & $\begin{array}{l}\text { CAHA } \\
\text { Asiago }\end{array}$ & $\begin{array}{l}1 \\
1\end{array}$ & Star in cluster \\
\hline HD 28446 & 043201.84 & +535439.1 & 5.77 & 0.10 & $\mathrm{~B} 0 \mathrm{III}+\mathrm{B} 0 \mathrm{IV} / \mathrm{V}$ & SCI & $\begin{array}{l}\text { CAHA } \\
\text { Asiago }\end{array}$ & $\begin{array}{l}2 \\
1\end{array}$ & Triple star (DL Cam) \\
\hline HD 43384 & 061658.71 & +234427.3 & 6.25 & 0.45 & B3Iab & PolStd & $\begin{array}{l}\text { CAHA } \\
\text { Asiago }\end{array}$ & $\begin{array}{l}2 \\
3\end{array}$ & Pulsating variable star \\
\hline HD 90508 & 102803.88 & +484705.7 & 6.43 & 0.60 & G0V & unPolStd & $\begin{array}{l}\text { CAHA } \\
\text { Asiago }\end{array}$ & $\begin{array}{l}1 \\
2\end{array}$ & Double star \\
\hline $\begin{array}{l}\text { HD } 39587 \\
\text { HD } 54439\end{array}$ & $\begin{array}{l}055422.98 \\
070823.20\end{array}$ & $\begin{array}{l}+201634.2 \\
-115108.6\end{array}$ & $\begin{array}{l}4.40 \\
7.68\end{array}$ & $\begin{array}{l}0.60 \\
0.05\end{array}$ & $\begin{array}{l}\text { G0VCH+M } \\
\text { B2/3II }\end{array}$ & $\begin{array}{l}\text { unPolStd } \\
\text { SCI }\end{array}$ & $\begin{array}{l}\text { Asiago } \\
\text { Asiago } \\
\text { VLT }\end{array}$ & $\begin{array}{l}2 \\
1 \\
1 \text { free }+1 \text { GG435 }\end{array}$ & Variable of RS CVn type \\
\hline $\begin{array}{l}\text { HD } 14357 \\
\text { HD } 21291\end{array}$ & $\begin{array}{lll}02 & 21 & 10.44 \\
03 & 29 & 04.13\end{array}$ & $\begin{array}{l}+565156.4 \\
+595625.2\end{array}$ & $\begin{array}{l}8.52 \\
4.22\end{array}$ & $\begin{array}{l}0.31 \\
0.41\end{array}$ & $\begin{array}{l}\text { B2III } \\
\text { B9Ia }\end{array}$ & $\begin{array}{l}\text { SCI } \\
\text { PolStd }\end{array}$ & $\begin{array}{l}\text { Asiago } \\
\text { Asiago }\end{array}$ & $\begin{array}{l}1 \\
1\end{array}$ & $\begin{array}{l}\text { Star in cluster } \\
\text { Pulsating variable star }\end{array}$ \\
\hline HD 73420 & 083637.12 & -440448.2 & 8.85 & 0.07 & B2III/III & SCI & VLT & 1 free + 1 GG435 & \\
\hline HD 78785 & 090824.09 & -461513.3 & 8.60 & 0.51 & B2III & SCI & VLT & 1 free +2 GG435 & \\
\hline HD 96042 & 110340.56 & -592559.1 & 8.23 & 0.18 & B1(V)ne & SCI & VLT & 2 free +2 GG435 & Emission-line star \\
\hline HD 141318 & 155106.80 & -550319.9 & 5.77 & -0.01 & B2III & SCI & VLT & 2 free +2 GG435 & Pulsating variable star \\
\hline HD 152245 & 165400.48 & -403158.2 & 8.37 & 0.13 & B0Ib & SCI & VLT & 1 free +2 GG435 & Star in cluster \\
\hline HD 152853 & 165807.93 & -455856.5 & 7.94 & 0.11 & B2III & SCI & VLT & 1 free +1 GG435 & Star in cluster \\
\hline
\end{tabular}

Notes. The coordinates, brightness and spectral type were taken from the SIMBAD Astronomical Database. Type indicates if the star is a polarized standard star (PolStd), unpolarized standard star (unPolStd), or one of our science targets (SCI). No. of Epochs is the number of epochs observed with a particular instrument: FORS2 (VLT), CAFOS (CAHA), or AFOSC (Asiago).

(FORS2) in spectropolarimetric mode (PMOS) mounted on the UT1 Cassegrain focus of the Very Large Telescope (VLT) in Chile; the Asiago Faint Object Spectrograph and Camera (AFOSC) mounted at the $1.82 \mathrm{~m}$ Copernico telescope at the Asiago Observatory in northern Italy; and the Calar Alto Faint Object Spectrograph (CAFOS) mounted at the Calar Alto $2.2 \mathrm{~m}$ telescope in Andalusia, Spain.

The characteristics of the instruments and corresponding differences in the data reduction are described in the following subsections.

\subsection{FORS2 at the VLT}

FORS2 in PMOS mode is a dual-beam polarimeter. The spectrum produced by the grism is split by the Wollaston prism into two beams with orthogonal directions of polarization: ordinary (o) and extraordinary (e) beam. The data used in this work were obtained with the $300 \mathrm{~V}$ grism, with and without the GG435 filter, and with the half-wave retarder plate positioned at angles of $0^{\circ}, 22.5^{\circ}, 45^{\circ}$, and $67.5^{\circ}$ (Program ID: 094.C-0686). The half-wave retarder plate angle is measured between the acceptance axis of the ordinary beam of the Wollaston prism (which is aligned to the north-south direction) and the fast axis of the retarder plate.

The data were reduced using standard procedures in IRAF. Wavelength calibration was achieved using He-Ne-Ar arc lamp exposures. The typical RMS accuracy is $\sim 0.3 \AA$. The data were bias subtracted, but not flat-field corrected. However, the effects of improper correction were minimized by taking advantage of the redundant number of half-wave positions (see Patat \& Romaniello 2006).
Ordinary and extra-ordinary beams were extracted in an unsupervised way using the PyRAF apextract.apall procedure, with a fixed aperture size of 10 pixels. The synthetic broadband polarization degree was computed by integrating the total flux weighted with Bessel's BVRI passband filters. We binned the spectra in $50 \AA$ bins, in order to obtain a higher signal-tonoise ratio $(\mathrm{S} / \mathrm{N})$, and calculated the Stokes parameters $Q$ and $U$, polarization degree $P$, and polarization angle $\theta_{P}$ as a function of wavelength.

The Stokes parameters $Q$ and $U$ were derived via Fourier transformation, as described in the FORS2 User Manual (ESO 2015):

$Q=\frac{2}{N} \sum_{i=0}^{N-1} F\left(\theta_{i}\right) \cos \left(4 \theta_{i}\right)$

$U=\frac{2}{N} \sum_{i=0}^{N-1} F\left(\theta_{i}\right) \sin \left(4 \theta_{i}\right)$

where $F\left(\theta_{i}\right)$ are the normalized flux differences between the ordinary $\left(f^{o}\right)$ and extra-ordinary $\left(f^{e}\right)$ beams:

$F\left(\theta_{i}\right)=\frac{f^{o}\left(\theta_{i}\right)-f^{e}\left(\theta_{i}\right)}{f^{o}\left(\theta_{i}\right)+f^{e}\left(\theta_{i}\right)}$

at different half-wave retarder plate position angles $\theta_{i}=i * 22.5^{\circ}$.

Although FORS2 is equipped with a super-achromatic halfwave plate, residual retardance chromatism is present. The wavelength dependent retardance offset $(\Delta \theta(\lambda))$ is tabulated in the FORS2 User Manual. The chromatism was corrected through the following rotation of the Stokes parameters:

$Q_{0}=Q \cos 2 \Delta \theta(\lambda)-U \sin 2 \Delta \theta(\lambda)$

$U_{0}=Q \sin 2 \Delta \theta(\lambda)+U \cos 2 \Delta \theta(\lambda)$ 
Finally we calculated the polarization:

$P=\sqrt{Q^{2}+U^{2}}$

and the polarization angle:

$\theta_{0}=\frac{1}{2} \arctan \left(U_{0} / Q_{0}\right)$

The reliability of data obtained with FORS2 is demonstrated in Cikota et al. (2017a). They used archival data of polarized and unpolarized stars to test the stability and capabilities of the spectropolarimetric mode (PMOS) of the FORS2 instrument, and found a good temporal stability since FORS2 was commissioned, and a good observational repeatability of total linear polarization measurements with an RMS $\lesssim 0.21 \%$. Cikota et al. (2017a) also found a small $(\lesssim 0.1 \%)$ instrumental polarization and fit linear functions to correct Stokes $Q$ and $U$, which we applied to the FORS2 data in this work.

\subsection{AFOSC at the $1.82 \mathrm{~m}$ Copernico telescope}

Spectropolarimetry with AFOSC was obtained using a simple combination of two Wollaston prisms and two wedges, a grism, and a slit mask of 2.5 arcsec wide and 20 arcsec long slitlets. This configuration permits measurements of the polarized flux at four polarimetric channels simultaneously, that is, at angles 0 , 45, 90 and 135 degrees, without the need of a half-wave retarder plate (Oliva 1997). For any given rotator adapter angle $\theta_{i}$, four fluxes can be measured. We group them into two groups, which we call ordinary $(\mathrm{O})$ and extraordinary $(\mathrm{E})$. We indicate them as $f_{O 1, i}, f_{E 1, i}$ and $f_{O 2, i}, f_{E 2, i}$. We used them to indicate the generic four beams $f_{0}, f_{90}$ and $f_{45}, f_{135}$, respectively.

However, in order to remove possible instrumental problems (i.e., imperfect beam splitting, flat fielding, etc.) it is convenient to obtain at least two sets of data. This can be achieved by rotating the instrument by 90 degrees with respect to the sky, so that a pair-wise swap between the corresponding polarimeter channels, 0 to 90 and 45 to 135 degrees, is performed.

The final $Q$ and $U$ were obtained via the Fourier approach:

$Q=\frac{1}{N} \sum_{i=0}^{N-1}\left(F_{1, i} \cos \left(2 \theta_{i}\right)-F_{2, i} \sin \left(2 \theta_{i}\right)\right)$

$U=\frac{1}{N} \sum_{i=0}^{N-1}\left(F_{1, i} \sin \left(2 \theta_{i}\right)+F_{2, i} \cos \left(2 \theta_{i}\right)\right)$,

where $\mathrm{N}$ is the number of rotator adapter angles, $\theta_{i}=\frac{\pi}{4} i$, and $F_{1, i}$ and $F_{2, i}$ are normalized flux ratios:

$F_{1, i}=\frac{f_{O 1, i}-f_{E 1, i}}{f_{O, 1, i}+f_{E, i}}$

$F_{2, i}=\frac{f_{O 2, i}-f_{E 2, i}}{f_{O 2, i}+f_{E 2, i}}$

Finally, we calculated the polarization degree and angle as given in Eqs. (5) and (6) respectively.

\subsection{CAFOS at the Calar Alto $2.2 \mathrm{~m}$ telescope}

CAFOS is a dual-beam polarimeter, similar to FORS2 in PMOS mode, composed of a half-wave retarder followed by a Wollaston prism that splits the incoming beam into an ordinary and an extra-ordinary beam. The data processing is as described for FORS2 in Sect. 3.1.

The CAFOS instrument was characterized in Patat \& Taubenberger (2011). They used polarized standard starsto quantify the HWP chromatism, which causes a peak-to-peak oscillation of $\sim 11$ degrees. From observations of unpolarized standard stars, they found an instrumental polarization likely produced by the telescope optics, which appears to be additive. The instrumental polarization is $\sim 0.3 \%$ between $4000 \AA$ and $8600 \AA$, and grows to $\sim 0.7 \%$ below $4000 \AA$. It can be removed by subtracting the instrumental components in the $Q-U$ Stokes plane. After correcting for the HWP chromatism and instrumental polarization, Patat \& Taubenberger (2011) concluded that an accuracy of $\sim 0.1 \%$ can be reached with four HWP angles and a sufficient $\mathrm{S} / \mathrm{N}$.

\section{Data processing and results}

The data were obtained with FORS2 in eight different nights between 2014-10-10 and 2015-02-06 (Program ID: 094.C-0686), with CAFOS in the night of 2015-04-29, and with AFOSC in five nights at three observing runs starting on 2015-02-09, 201503-09, and 2016-08-02.

\subsection{Standard stars}

We investigate the accuracy and reliability of the instruments using unpolarized and polarized standard stars. Cikota et al. (2017a) used archival data of eight unpolarized standard stars observed at 40 epochs between 2009 and 2016 to test the stability and capabilities of the spectropolarimetric mode (PMOS) of the FORS2 instrument. They showed that the polarization degree and angle are stable at the level of $\lesssim 0.1 \%$ and $\lesssim 0.2$ degrees, respectively. They found a small $(\lesssim 0.1 \%)$ wavelengthdependent instrumental polarization and derived linear functions for the Stokes $Q$ and $U$, which we applied to the observed Stokes parameters. In this paper, we therefore focus on unpolarized and polarized standard stars observed with CAFOS and AFOSC.

Two unpolarized standard stars (HD 144579, and HD 90508), and two polarized standard stars (HD 154445 and HD 43384) were observed with CAFOS. We did not find any significant instrumental polarization in the CAFOS observations, and the polarization values are consistent with the literature. The results are given in Sect. A.1.

We used observations of three unpolarized standard stars to investigate possible instrumental polarization of AFOSC: HD 90508, HD 39587 and HD 144579; and three polarized standard stars to test the reliability: HD 43384, HD 21291, and HD 198478. We did not detect any significant instrumental polarization, but the polarization degrees of polarized stars observed at different epochs vary by $\sim 0.3 \%$. The inconsistencies might be caused by diffraction of light from the edge of the slit (see Keller C.U. in Trujillo-Bueno et al. 2002, p. 303) or by an inaccuracy of the instruments rotation angle (see Bagnulo et al. 2017). The results are given in Sect. A.2.

\subsection{FORS2 science data}

FORS2 is the most stable instrument used in this work, and we are confident that the data gained with FORS2 are accurate and can be used as reference for comparison to other instruments (see Cikota et al. 2017a). Eight stars with anomalous extinction sightlines were observed with FORS2 (see Table 1). HD 54439 was also observed with AFOSC, and HD 137569 was observed with all three instruments, FORS2, CAFOS, and AFOSC, which we briefly discuss in this section. HD 78785, 


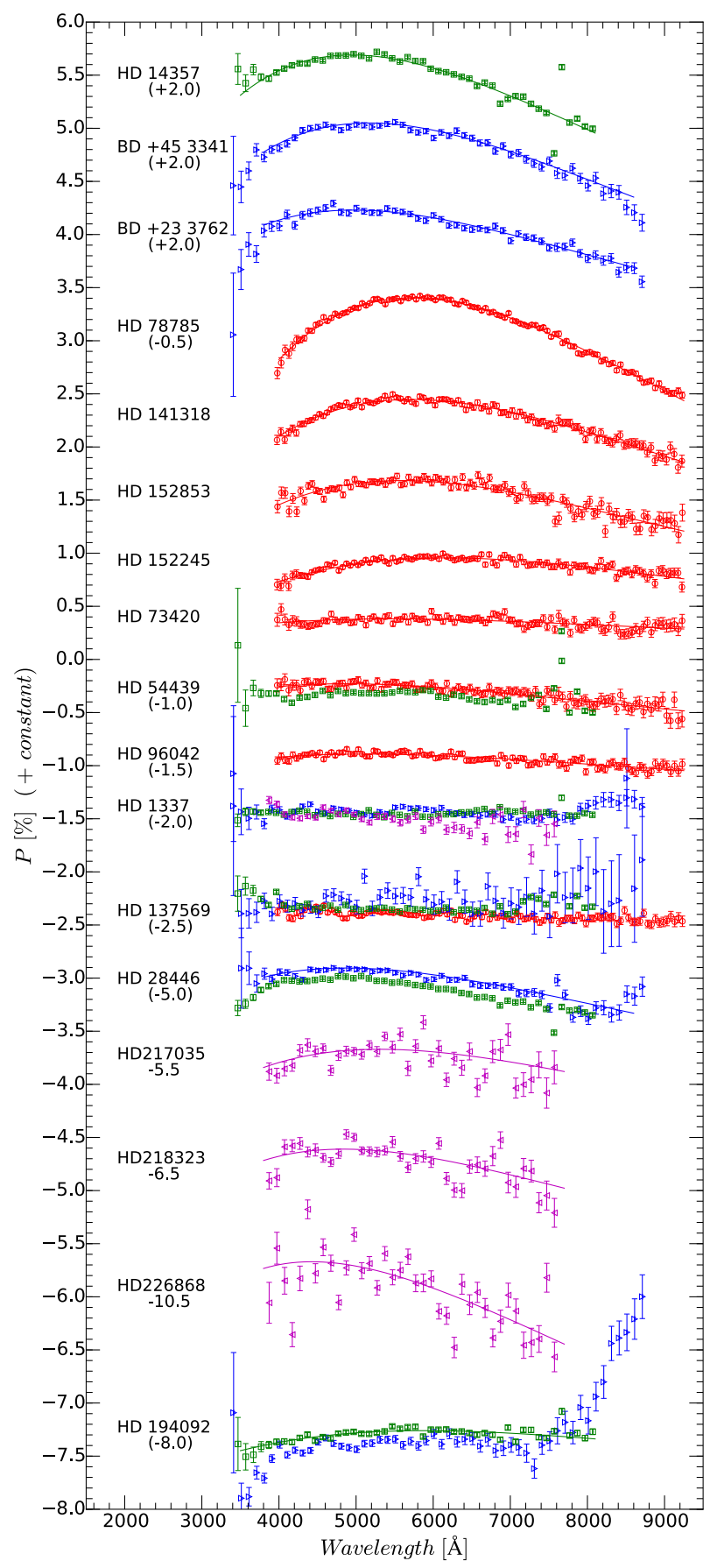

Fig. 1. Weighted averages of observed polarization curves for all science targets, derived with different instruments. The red circles show observations performed with FORS2, blue triangles with CAFOS, green squares with AFOSC, and left-pointing purple triangles with HPOL. The full lines denote the Serkowski fits, as parametrized in Table 3.

HD 141318, HD 152853, HD 152245, HD 73420, and HD 96042 were observed only with FORS2.

We extracted the spectra and calculated the polarization dependencies as described in Sect. 3.1. The correction for the instrumental polarization determined in Cikota et al. (2017a) was also applied to Stokes $Q$ and $U$. The targets have been observed with and without the GG435 filter. The GG435 filter blocks the blue light and thus prevents the second order spectrum. However, the effect in polarization is very small, and significant only for very blue spectral energy distributions and when measuring line polarization (Patat et al. 2010). For our reddened targets, the second-order polarization is negligible. Therefore, for wavelengths $\lambda>4250 \AA$, we calculated the weighted mean of all epochs, and for wavelengths $\lambda<4250 \AA$, we calculated the weighted mean of all epochs taken without the GG435 filter. We then merged both ranges to one polarization spectrum and parameterized it by fitting a Serkowski curve (Eq. (1)) to the data. The individual results can be found in Table C.1. The polarization dependencies are shown in Fig. 1, and the Serkowski parameters are given in Table 3.

HD 137569 is an interesting case because it has relatively high reddening $E(B-V) \sim 0.40 \mathrm{mag}$ (Mazzei \& Barbaro 2011), but its polarization degree is consistent with zero (Fig. 1). Its mean Stokes $Q$ and $U$ are $-0.07 \pm 0.05 \%$ and $0.01 \pm 0.03 \%$. HD 137569 was also observed with CAFOS and AFOSC, and the results are consistent with the FORS2 observations. Furthermore, HD 137569 is a spectroscopic binary with a period of 529.8 days, and shows observational signatures normally seen in post-AGB stars (Giridhar \& Arellano Ferro 2005). This is interesting, because sightlines to post-AGB stars usually show continuum polarization (Johnson \& Jones 1991).

\subsection{CAFOS science data}

Six stars with anomalous extinction sightlines were observed with CAFOS. HD 1337 and HD 28446 were also observed with AFOSC, and HD 137569 was additionally observed with both FORS2 and AFOSC (see also Sect. 4.2). BD +453341, BD+23 3762, and HD 194092 were observed only with CAFOS.

After the beam extraction using the apextract.apall procedure of PyRAF, we binned the spectra into $100 \AA$ wide bins and calculated the polarization as described in Sect. 3.3. Finally, we fit the Serkowski curve in the range between 3800-8600. . The individual results are listed in Table C.2. The polarization dependencies are shown in Fig. 1, and the Serkowski parameters are listed in Table 3. Below we discuss the interesting cases.

\subsubsection{HD 1337}

HD 1337 is a close spectroscopic binary star, with a period of 3.52 days (Pourbaix et al. 2004), and may be surrounded by a common-envelope, where significant dust amounts may be produced (Lü et al. 2013). HD 1337 has a constant polarization degree of $\sim 0.55 \%$. The CAFOS observations are also consistent with the AFOSC observations, which confirms the polarization wavelength independence (see Fig. 1). In this case, the Serkowski fit is not meaningful.

\subsubsection{HD 194092}

For HD 194092, the polarization observed by CAFOS follows a Serkowski curve until $7250 \AA$, when it starts to steeply increase from $p \sim 0.5 \%$ to $p \sim 2 \%$ at $8650 \AA$. The steep increase is not present in the AFOSC observations and is an artifact that we cannot explain. Thus, we fit the Serkowski curve in the range from $3800-7250 \AA$, and find $\lambda_{\max }=5728 \pm 235 \AA, p_{\max }=$ $0.64 \pm 0.01 \%$ and $K=1.46 \pm 0.47$.

\subsection{AFOSC science data}

Six stars with anomalous extinction sightlines have been observed with AFOSC. HD 28446, HD 1337, and HD 194092 
were also observed with CAFOS and HD 54439 with FORS2, while HD 137569 was additionally observed with CAFOS and FORS2 (see also Sect. 4.2). HD 14357 was observed only with AFOSC.

We extracted the beams from 3400-8150 A using the same standard procedures in IRAF as for the extraction of FORS 2 and CAFOS spectra, binned the data to $100 \AA$ wide bins, and calculated the polarization as described in Sect. 3.2. The polarization spectra from $3500-8150 \AA$ were fitted with a Serkowski curve, excluding the range from $7500-7700 \AA$, which is contaminated by the telluric $\mathrm{O}_{2}$ line.

The unpolarized standard stars are consistent with zero, which implies that there is no significant instrumental polarization (Sect. A.2). However, based on the measurements of the polarized standard stars HD 43384 and HD 21291 (see Sect. A.2), which show a negative offset compared to the literature values, we conclude that the accuracy of the polarization measurements is within $\sim 0.4 \%$. HD 28446 and HD 54439 show an offset of $\sim-0.1 \%$ compared to the results achieved with CAFOS and FORS2, respectively, while the measurements of HD 1337 are consistent with the CAFOS measurements, and the measurements of HD 137569 are consistent with the FORS2 and CAFOS measurements (Fig. 1). The individual results are listed in Table C.3.

HD 14357 is the only AFOSC target that has no common observations with another instrument. From the Serkowski fit, we determined $\lambda_{\max }=4942 \pm 31 \AA, p_{\max }=3.69 \pm 0.01 \%$ and $K=0.91 \pm 0.04$. Based on HD 43384 and other polarized stars, the $\lambda_{\max }$ and $K$ values are probably accurate, while there might be a negative offset, $\lesssim 0.4 \%$, to the true value of $p_{\max }$.

\subsection{HPOL science data}

We found archival data for HD 1337 (which was also observed with CAFOS and AFOSC), and three additional stars of the Mazzei \& Barbaro (2011) sample in the University of Wisconsin's Pine Bluff Observatory (PBO) HPOL spectropolarimeter (mounted at the $0.9 \mathrm{~m} \mathrm{f} / 13.5$ cassegrain) data set. All targets were observed prior to the instrument update in 1995 , when HPOL was providing spectropolarimetry over the range of $3200 \AA$ to $7750 \AA$, with a spectral resolution of $25 \AA$. A halfwave plate was rotated to eight distinct angles to provide the spectropolarimetric modulation (Wolff et al. 1996).

The HPOL data are available in the Mikulski Archive for Space Telescopes (MAST) at the Space Telescope Science Institute. They include the Stokes parameters $Q$ and $U$, and the error as a function of wavelength.

We calculated the polarization and polarization angle using Eqs. (5) and (6), and fit a Serkowski curve to the data (Eq. (1)). The results are given in Table 3.

\subsection{Literature science data}

We found polarization measurements in the literature (Coyne et al. 1974; Serkowski et al. 1975) of eight stars of the Mazzei \& Barbaro (2011) sample. Three of the stars have been observed in this work with FORS2, AFOSC, or CAFOS, and for two stars, we found archival HPOL data. The stars were observed with broadband polarimeters and were characterized by fitting the Serkowski curve (Table 2). However, because the authors assumed a fixed $K=1.15$, we could only partially use the measurements from Coyne et al. (1974) and Serkowski et al. (1975) in our further analysis.
Table 2. $\lambda_{\max }$ and $P_{\max }$ from the literature.

\begin{tabular}{|c|c|c|c|}
\hline Name & $\begin{array}{c}\lambda_{\max } \\
(\AA)\end{array}$ & $\begin{array}{c}P_{\max } \\
(\%)\end{array}$ & Reference \\
\hline $\mathrm{HD} 2$ & 4900 & 0.32 & Coy \\
\hline HD & 00 & 1.63 & Coy \\
\hline HD & $5900 \pm$ & $2.13 \pm$ & Serkowski et al. (1975 \\
\hline HD $28446^{b, c}$ & $5500 \pm 100$ & $2.01 \pm 0.10$ & Coyne et al. (1974) \\
\hline HD $226868^{d}$ & $5000=$ & 5.04 & Coyne et a \\
\hline HD $218323^{d}$ & $5200 \pm 200$ & $1.95 \pm 0.14$ & Coyn \\
\hline HD $78785^{a}$ & $5800 \pm 224$ & $4.05 \pm 0.04$ & Serkowski et al. (19' \\
\hline HD $141318^{a}$ & $5700 \pm 100$ & $2.42 \pm 0.08$ & Serkowski et al. (1975) \\
\hline
\end{tabular}

Notes. ${ }^{(a)}$ Also observed with FORS2. ${ }^{(b)}$ Also observed with AFOSC. ${ }^{(c)}$ Also observed with CAFOS. ${ }^{(d)}$ Also observed with HPOL.

Table 3. Final Serkowski parameters.

\begin{tabular}{|c|c|c|c|c|}
\hline \multirow[b]{2}{*}{ Name } & \multirow[b]{2}{*}{ Telescope } & \multicolumn{3}{|c|}{ Serkowski parameters } \\
\hline & & $\begin{array}{l}\lambda_{\max } \\
(\AA)\end{array}$ & $\begin{array}{l}p_{\max } \\
(\%)\end{array}$ & $K$ \\
\hline HD $137569^{a}$ & VLT & & $\sim 0.1$ & \\
\hline $\mathrm{HD}$ & VLT & $4859 \pm 129$ & $0.77 \pm 0.01$ & 0.97 \\
\hline In & VLT & $5465 \pm 175$ & $0.38 \pm 0.01$ & $1.04 \pm 0.21$ \\
\hline HD & VLT & $5732 \pm 8$ & 3.90 & 1.25 \\
\hline & VLT & $5109 \pm 124$ & $0.61=$ & 0.84 \\
\hline HD 1 & VLT & $5719 \pm 17$ & $2.45 \pm 0.01$ & $1.19 \pm 0.03$ \\
\hline HD 1 & VLT & $6169 \pm 33$ & 0.96 & 0.07 \\
\hline HD 1 & VLT & $5584 \pm 46$ & 1.69 & 1.30 \\
\hline $\mathrm{BD}+$ & CAHA & $4965 \pm 61$ & 2.23 & 0.92 \\
\hline $\mathrm{BD}+453341$ & CAHA & $5166 \pm 31$ & $3.05 \pm 0.01$ & $1.00 \pm 0.05$ \\
\hline HD $1337^{a}$ & CAHA & $\ldots$ & $0.55 \pm 0.01$ & \\
\hline HD 28446 & CAHA & $4865 \pm 76$ & $2.10 \pm 0.01$ & 0.7 \\
\hline HD 19 & Asiago & $5884 \pm 107$ & 0.74 & $1.09 \pm 0.18$ \\
\hline HD 14 & Asiago & $4942 \pm 31$ & $3.69 \pm 0.01$ & $0.91 \pm 0.04$ \\
\hline HD 226868 & HPOL & $4425 \pm 262$ & $4.83 \pm 0.04$ & $0.57 \pm 0.14$ \\
\hline HD 2 & HPOL & $4837 \pm 128$ & $1.89 \pm 0.01$ & $1.00 \pm 0.20$ \\
\hline HD 217035 & HPOL & $5309 \pm 126$ & $1.83 \pm 0.02$ & $0.88 \pm 0.23$ \\
\hline
\end{tabular}

Notes. ${ }^{(a)}$ HD 137569 and HD 1337 have constant polarization curves that could not be fit with a Serkowski curve.

\section{Data analysis}

Figure 2 shows the sample of 15 anomalous sightlines (listed in Table 3, excluding HD 137569 and HD 1337), compared to a sample of Galactic stars observed by Whittet et al. (1992) and a sample SNe Ia from Patat et al. (2015) and Zelaya et al. (2017; see Appendix B).

Mazzei \& Barbaro (2011) used models of Weingartner \& Draine (2001, hereafter WD01) and the updates by Draine \& Li (2007) to compute grain-size distributions for spherical grains of amorphous silicate and carbonaceous grains consisting of graphite grains and polycyclic aromatic hydrogenated (PAH) molecules. As described in Mazzei \& Barbaro (2011), they bestfit the extinction curves with models as described above (Sect. 2) to derive the properties of the dust in terms of dust-to-gas ratios, abundance ratios, and small-to-large grain size ratios of carbon and of silicon. They excluded HD 1337 (a W UMa type variable) and HD 137569 (a post-AGB star) from their analysis before performing the modeling because of the extremely low CCM $R_{V}$ 


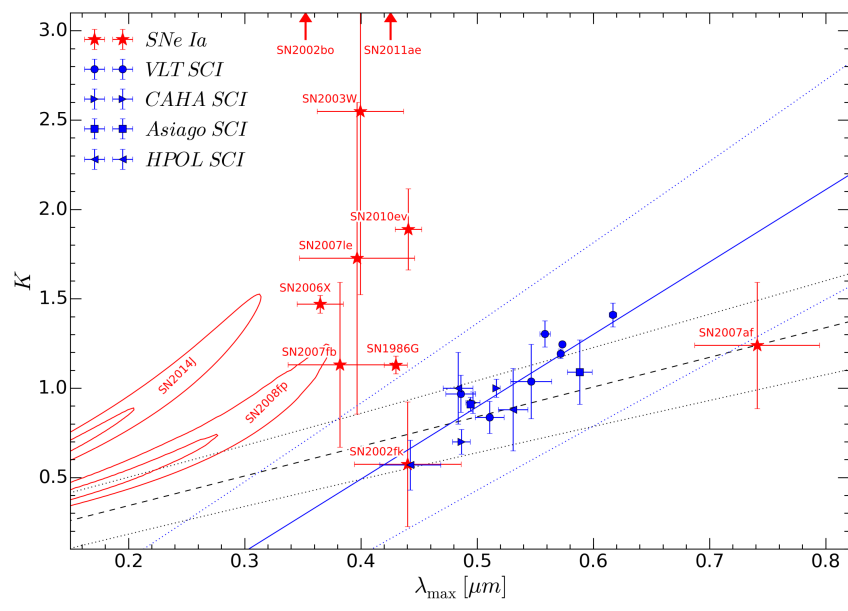

Fig. 2. Stars with anomalous extinction sightlines in the $\lambda_{\max }-\mathrm{K}$ plane. Blue shows stars with anomalous sightlines observed with FORS2 (circles), CAHA (right pointing triangles), AFOSC (squared), and HPOL (left-pointing triangles). The solid blue line represents the linear best fit to the sample, and its $1 \sigma$ deviation (dotted). The dashed black line traces the Whittet et al. (1992) relation and its $3 \sigma$ uncertainty (dotted). For comparison, a sample of SNe Ia from Patat et al. (2015) and Zelaya et al. (2017; see Appendix B) are marked with stars and red contours, which indicate the 10 and $20 \sigma$ confidence levels for SN 2008fp and SN 2014J.

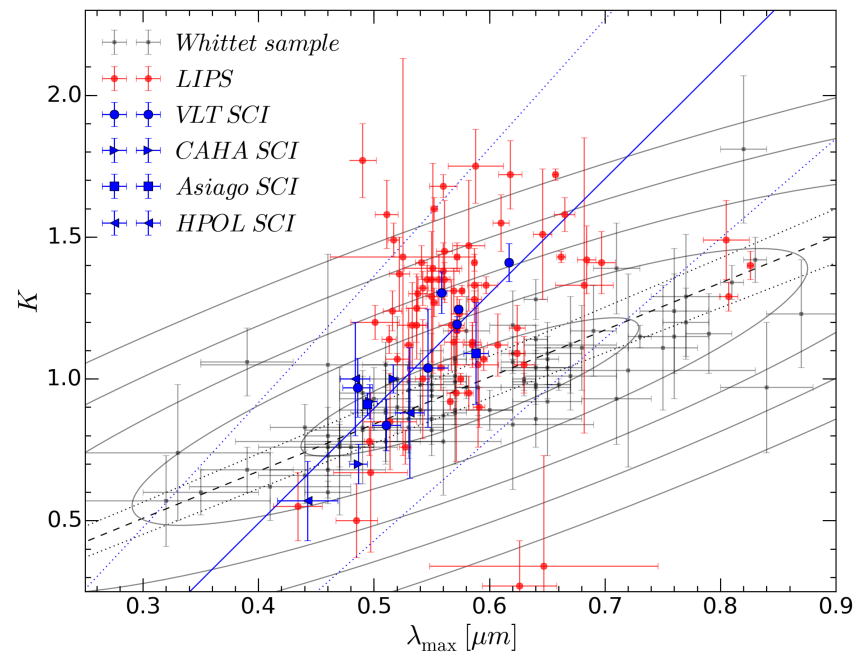

Fig. 3. Comparison of our sample of stars with anomalous extinction sightlines (blue symbols) to the Whittet et al. (1992) sample (gray squares) and the LIPS sample (Bagnulo et al. 2017, red circles). The gray ellipses represent $1-5 \sigma$ confidence levels for the Whittet et al. (1992) sample. The dashed black line traces the Whittet et al. (1992) relation and its $1 \sigma$ uncertainty (dotted).

values of these sightlines, $R_{V} \approx 0.6$ and $R_{V} \approx 1.1$, respectively, which are well outside the range explored by CCM extinction curves.

We combined the $\lambda_{\max }$ values from this work with the results of WD01 best-fit models of Mazzei \& Barbaro (2011, their Table 4), and computed the Pearson correlation coefficient, $\rho$, and the $p$-value for testing the non-correlation between $\lambda_{\max }$ and their derived dust-to-gas ratio $\left(\frac{\rho_{d}}{\rho_{\mathrm{H}}}\right)$, carbon and silicon abundances compared to solar values $\left(\frac{\mathrm{C}}{\mathrm{C}_{\odot}}, \frac{\mathrm{Si}}{\mathrm{Si}_{\odot}}\right), R_{V}$ value, the ratio between reddening and total hydrogen column density $\left(\frac{E(B-V)}{N_{\mathrm{H}}}\right)$,

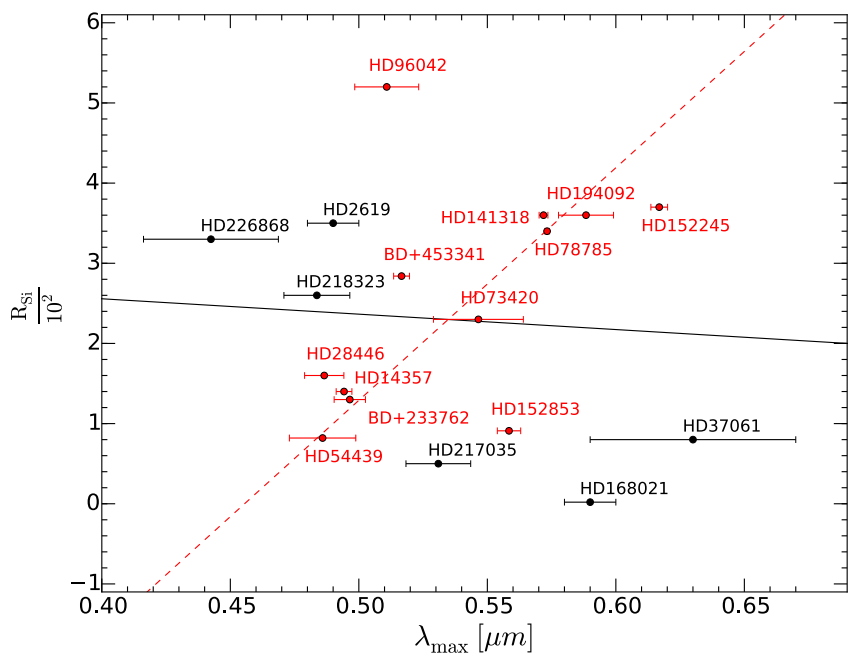

Fig. 4. $R_{\mathrm{Si}}-\lambda_{\max }$ relationship. The red dots represent stars observed with FORS2, AFOSC, and CAFOS, and the black dots show seven additional measurements from HPOL or from the literature. The red dashed line is the linear least-squares fit to the red dots, and the black solid line is the linear least-squares fit to all data.

and small-to-large grain size ratios of carbon $\left(R_{\mathrm{C}}\right)$ and silicon $\left(R_{\mathrm{Si}}\right)$. They considered grains as small if their size is $\leqslant 0.01 \mu \mathrm{m}$, and otherwise as large. We note that a detailed analysis and discussion of the WD01 best-fit model results for the whole sample of 64 anomalous sightlines is given in Mazzei \& Barbaro (2011).

The strongest correlation we found between $\lambda_{\max }$ and $R_{\mathrm{Si}}$, with a correlation factor of $\rho=0.50$, and the p-value for testing non-correlation of $p=0.10$, while there is no correlation between $\lambda_{\max }$ and other parameters $(\rho \lesssim 0.25)$. We also added three stars observed by HPOL and three stars from the literature (Coyne et al. 1974; Serkowski et al. 1975) to the sample and repeated the same correlation tests. These additional stars have anomalous extinction sightlines (Mazzei \& Barbaro 2011), but higher $R_{V}$ values than our observed sample (see Table 4). After including the three HPOL observations and three stars from literature, the $R_{\mathrm{Si}}-\lambda_{\max }$ correlation factor drops to $\rho=-0.06$. The $R_{\mathrm{Si}}-\lambda_{\max }$ correlation is shown in Fig. 4. However, these results should be taken with care because when we fit the data, we assumed no uncertainty in $R_{\mathrm{Si}}$. This may be problematic because the realistic uncertainty in the model quantities should be considerable, probably larger than in $\lambda_{\max }$, which is a simple measurement. In this way, the fit is strongly driven by the few stars with very low $\lambda_{\max }$ errors. The relationship is further discussed in Sect. 7.7.

\section{Dust properties inferred from simulations}

The properties of dust grains toward the considered stars were obtained in Mazzei \& Barbaro (2008), where the authors performed theoretical model fitting to the anomalous extinction curves. The authors found that to reproduce the anomalous extinction, silicate grains must be concentrated in small sizes of $a<0.1 \mu \mathrm{m}$ (see also Mazzei \& Barbaro 2011). Such small silicate grains cannot reproduce the normal $\lambda_{\max }$ that is measured. In this section, we therefore infer the essential dust properties (i.e., size distribution and alignment) by fitting both extinction curves with low $R_{V}$ values and normal polarization curves. The results are used to interpret the $\lambda_{\max }-K$ relationship, the deviation of $K$ from the average value from a sample of "normal" Galactic stars, and to test the relationship between $R_{\mathrm{Si}}$ and $\lambda_{\max }$. 


\subsection{Dust model and observational constrains}

\subsubsection{Dust model: size distribution}

We adopted a mixed-dust model consisting of astronomical silicate and carbonaceous grains (see Weingartner \& Draine 2001, hereafter WD01). The same size distribution model was also used in Mazzei \& Barbaro (2011). We assumed that grains have oblate spheroidal shapes, and let $a$ be the effective grain size defined as the radius of the equivalent sphere with the same volume as the grain.

Following WD01, the grain size distribution of dust component $j$ is described by an analytical function:

$\frac{d n_{j}}{n_{\mathrm{H}} d a}=D_{j}(a)+\frac{C_{j}}{a}\left(\frac{a}{a_{t, j}}\right)^{\alpha_{j}} F\left(a ; \beta_{j}, a_{t, g}\right) G\left(a ; a_{t, j}, a_{c, j}\right)$,

where $a$ is the grain size, $j=$ sil, carb for silicate and carbonaceous compositions, $D_{j}(a)$ is the size distribution for very small grains, $a_{t, j}, a_{c, j}$ are model parameters, and $C_{j}$ is a constant determined by the total gas-to-dust mass ratio.

The coefficients $F$ and $G$ read

$F\left(a ; \beta_{j}, a_{t, j}\right)=1+\beta_{j} a / a_{t, j}$ for $\beta_{j}>0$,

$F\left(a ; \beta_{j}, a_{t, j}\right)=\left(1-\beta_{j} a / a_{t, j}\right)^{-1}$ for $\beta_{j}<0$,

and

$G\left(a ; a_{t, j}, a_{c, j}\right)=1$ for $a<a_{t, j}$,

$G\left(a ; a_{t, j}, a_{c, j}\right)=\exp \left(-\left[\left(a-a_{t, j}\right) / a_{c, j}\right]^{3}\right)$ for $a>a_{t, j}$.

The term $D_{j}=0$ for $j=$ sil. For very small carbonaceous grains (i.e., PAHs), $D_{j}(a)$ is described by a log-normal size distribution containing a parameter $b_{C}$ that denotes the fraction of $\mathrm{C}$ abundance present in very small sizes (see WD01 for more detail). Thus, the grain size distribution is completely described by a set of 11 parameters: $\alpha_{j}, \beta_{j}, a_{t, j}, a_{c, j}$, and $C_{j}$, where $j=$ sil, carb for silicate and carbonaceous compositions, and $b_{C}$.

\subsubsection{Dust model: alignment function}

Let $f_{\text {ali }}$ be the fraction of grains that are perfectly aligned with the symmetry axis $\hat{a}_{1}$ along the magnetic field $B$. The fraction of grains that are randomly oriented is thus $1-f_{\text {ali }}$. To parameterize the dependence of $f_{\text {ali }}$ on the grain size, we introduce the following function:

$f_{\mathrm{ali}}\left(a ; a_{\mathrm{ali}}, f_{\min }, f_{\max }\right)=f_{\min }+\left[1-\exp \left(-\frac{a}{a_{\mathrm{ali}}}\right)^{3}\right]\left(f_{\max }-f_{\min }\right)$,

where $a_{\text {ali }}$ describes the minimum size of aligned grains, $f_{\max }$ describes the maximum degree of grain alignment, and $f_{\text {min }}$ accounts for some residual small degree of alignment of very small grains. This alignment function reflects the modern understanding of grain alignment, where large grains are efficiently aligned by radiative torques (see, e.g., Hoang \& Lazarian 2016) and small grains are weakly aligned by paramagnetic relaxation (Hoang et al. 2014).

\subsection{Extinction and polarization model}

\subsubsection{Extinction}

The extinction of starlight due to scattering and absorption by interstellar grains in units of magnitude is given by

$\frac{A(\lambda)}{N_{\mathrm{H}}}=1.086 \sum_{j=s i l, c a r b} \int_{a_{\min }}^{a_{\max }} C_{\mathrm{ext}}^{j}(a, \lambda)\left(\frac{d n_{j}}{d a}\right) d a$,

where $C_{\text {ext }}$ is the extinction cross-section, $a_{\min }$ and $a_{\max }$ are the lower and upper cutoffs of the grain size distribution, and $N_{\mathrm{H}}$ is the total gas column density along the sightline.

\subsubsection{Polarization}

Modeling the starlight polarization by aligned grains is rather complicated because it requires a detailed knowledge of the orientation of grains with the magnetic field and the magnetic field geometry along the line of sight. Specifically, a realistic modeling needs to take into account the nutation of the grain symmetry axis $\hat{\mathbf{a}}_{1}$ around the angular momentum $J$, the precession of $J$ around $B$, and the distribution function of the cone angle between $J$ and $B$ (Hong \& Greenberg 1980; see Voshchinnikov 2012 for a review). However, an analytical distribution function for the cone angle is not known for the popular alignment mechanism by radiative torques (see Lazarian et al. 2015 and Andersson et al. 2015 for latest reviews). Therefore we adopted a picket-fence (PF) alignment model to compute the polarization, as used in previous works (Kim \& Martin 1995; Draine \& Allaf-Akbari 2006; Draine \& Fraisse 2009; Hoang et al. 2013, 2014). The essence of the PF model is as follows.

First, the oblate grain is assumed to be spinning around the symmetry axis $\hat{\mathbf{a}}_{1}$ (i.e., having perfect internal alignment). The magnetic field $B$ is assumed to lie in the plane of the sky $\hat{\mathbf{x}} \hat{\mathbf{y}}$ with $B \| \hat{\mathbf{x}}$, and the line of sight is directed along $\hat{\mathbf{z}}$. Therefore, the polarization cross-section contributed by the perfectly aligned grains is $C_{x}-C_{y}=\left(C_{\|}-C_{\perp}\right) f_{\text {ali }}$, where $C_{\|}$and $C_{\perp}$ are the cross-section for the incident electric field parallel and perpendicular to the symmetry axis, respectively (see Hoang et al. 2013). Of $\left(1-f_{\text {ali }}\right)$ randomly oriented grains, the fraction of grains that are aligned with $\hat{\mathbf{x}}, \hat{\mathbf{y}}, \hat{\mathbf{z}}$ are equal, of $\left(1-f_{\text {ali }}\right) / 3$. The total polarization produced by grains with $\hat{\mathbf{a}}_{1} \| B$ is then $C_{x}-C_{y}=$ $\left(C_{\|}-C_{\perp}\right)\left(f_{\text {ali }}+\left(1-f_{\text {ali }}\right) / 3\right)$. The polarization by grains aligned with $\hat{\mathbf{a}}_{1} \| \hat{\mathbf{y}}$ is $\left(1-f_{\mathrm{ali}}\right) / 3\left(C_{\|}-C_{\perp}\right) / 3$. Thus, the total polarization cross-section is $C_{x}-C_{y}=\left(C_{\|}-C_{\perp}\right)\left[\left(1+2 f_{\text {ali }}\right)-\left(1-f_{\text {ali }}\right)\right] / 3=$ $C_{\text {pol }} f_{\text {ali }}$.

Because graphite grains are not aligned with the magnetic field (Chiar et al. 2006; Hoang \& Lazarian 2016), we assumed that only silicate grains are aligned, while carbonaceous grains are randomly oriented. Therefore, the degree of polarization of starlight due to differential extinction by aligned grains along the line of sight is computed by

$\frac{p(\lambda)}{N_{\mathrm{H}}}=\int_{a_{\min }}^{a_{\max }} \frac{1}{2} C_{\mathrm{pol}}^{\mathrm{sil}}(a, \lambda) f_{\mathrm{ali}}(a) \frac{d n_{\mathrm{sil}}}{d a} d a$,

where $C_{\text {pol }}^{\text {sil }}$ is the polarization cross-section of silicate oblate grains, and $f_{\text {ali }}$ is given by Eq. (14). Here we take $C_{\text {ext }}$ and $C_{\text {pol }}$ computed for different grain sizes and wavelengths from Hoang et al. (2013).

We note that magnetic fields may be varying for the different stars. However, we here do not attempt to infer a dust model for each specific sightline. Instead, we only attempt to infer the general features of dust size distribution and alignment functions for 
Table 4. Observational data and results of modeling

\begin{tabular}{|c|c|c|c|c|c|c|c|c|c|c|}
\hline \multirow[b]{2}{*}{ Name } & \multicolumn{5}{|c|}{ Mazzei \& Barbaro (2011) } & \multicolumn{3}{|c|}{ Wegner (2002) } & \multirow[b]{2}{*}{$\begin{array}{l}\lambda_{\max } \\
(\AA)\end{array}$} & \multirow[b]{2}{*}{$\lambda_{\max }$ Reference } \\
\hline & Sp. & $\begin{array}{l}E(B-V) \\
(\mathrm{mag})\end{array}$ & $\mathrm{CCM} R_{V}$ & $R_{V}$ & $\frac{R_{\mathrm{Si}}}{10^{2}}$ & Sp. & $\begin{array}{l}E(B-V) \\
(\mathrm{mag})\end{array}$ & $R_{V}$ & & \\
\hline HD 54439 & B2III & 0.28 & $2.13 \pm 0.41$ & 1.98 & 0.82 & B1V & 0.28 & 2.88 & $4859 \pm 129$ & This work (VLT) \\
\hline HD 73420 & B2II/III & 0.37 & $2.47 \pm 0.32$ & 2.24 & 2.3 & & & & $5465 \pm 175$ & This work (VLT) \\
\hline HD 78785 & B2II & 0.76 & $2.55 \pm 0.17$ & 2.29 & 3.4 & B2II & 0.67 & 3.08 & $5732 \pm 8.2$ & This work (VLT) \\
\hline HD 96042 & $09.5 \mathrm{~V}$ & 0.48 & $1.97 \pm 0.24$ & 1.87 & 5.2 & B1V & 0.41 & 3.05 & $5109 \pm 124$ & This work (VLT) \\
\hline HD 141318 & B2II & 0.30 & $1.95 \pm 0.18$ & 1.77 & 3.6 & & & & $5719 \pm 17$ & This work (VLT) \\
\hline HD 152245 & BOIII & 0.42 & $2.25 \pm 0.29$ & 2.02 & 3.7 & B0Ib & 0.31 & 2.95 & $6169 \pm 33$ & This work (VLT) \\
\hline HD 152853 & B2II/III & 0.37 & $2.50 \pm 0.33$ & 2.19 & 0.91 & & $\ldots$ & $\ldots$ & $5584 \pm 46$ & This work (VLT) \\
\hline $\mathrm{BD}+233762$ & B0.5III & 1.05 & $2.47 \pm 0.12$ & 2.15 & 1.3 & & $\ldots$ & $\ldots$ & $4965 \pm 61$ & This work (CAHA) \\
\hline $\mathrm{BD}+453341$ & B1II & 0.74 & $2.46 \pm 0.17$ & 2.22 & 2.84 & $\ldots$ & $\ldots$ & $\ldots$ & $5166 \pm 31$ & This work (CAHA) \\
\hline HD 28446 & B0III & 0.46 & $2.46 \pm 0.26$ & 2.20 & 1.6 & $\ldots$ & $\ldots$ & $\ldots$ & $4865 \pm 76$ & This work (CAHA) \\
\hline HD 194092 & B0.5III & 0.41 & $2.50 \pm 0.30$ & 2.18 & 3.6 & $\ldots$ & $\ldots$ & $\ldots$ & $5884 \pm 107$ & This work (Asiago) \\
\hline HD 14357 & B2II & 0.56 & $2.31 \pm 0.21$ & 2.12 & 1.4 & B1.5II & 0.49 & 2.88 & $4942 \pm 31$ & This work (Asiago) \\
\hline HD 226868 & $\mathrm{~B} 0 \mathrm{Ib}$ & 1.08 & $3.20 \pm 0.14$ & 2.78 & 3.3 & $\mathrm{~B} 0 \mathrm{Ib}$ & 1.03 & 3.32 & $4424.6 \pm 262.4$ & (This work, HPOL) \\
\hline HD 218323 & BOIII & 0.90 & $2.55 \pm 0.15$ & 2.30 & 2.6 & $\ldots$ & $\ldots$ & $\ldots$ & $4836.7 \pm 128.2$ & (This work, HPOL) \\
\hline HD 217035 & B0V & 0.76 & $2.77 \pm 0.18$ & 2.44 & 0.5 & & $\ldots$ & $\ldots$ & $5309.3 \pm 125.9$ & (This work, HPOL) \\
\hline HD 2619 & B0.5III & 0.85 & $2.55 \pm 0.15$ & 2.34 & 3.5 & $\ldots$ & $\ldots$ & $\ldots$ & $4900 \pm 100$ & Coyne et al. (1974) \\
\hline HD 37061 & B1V & 0.52 & $4.50 \pm 0.38$ & 3.82 & 0.8 & $\mathrm{~B} 0.5 \mathrm{~V}$ & 0.47 & 4.14 & $6300 \pm 400$ & Coyne et al. (1974) \\
\hline HD 168021 & B0Ib & 0.55 & $3.15 \pm 0.27$ & 2.74 & 0.02 & $\ldots$ & $\ldots$ & $\ldots$ & $5900 \pm 100$ & Serkowski et al. (1975) \\
\hline
\end{tabular}

Notes. Spectral type (Sp.), $E(B-V)$ and $\mathrm{CCM} R_{V}, R_{V}$, and $R_{\mathrm{Si}}$ are taken from Tables 1 and 4 of Mazzei \& Barbaro (2011). The Sp. and $E(B-V)$ values in Mazzei \& Barbaro (2011) are taken from Savage et al. (1985). CCM $R_{V}$ is determined by fitting the IR observations with the CCM extinction curve, while $R_{V}$ is determined from the best-fit of WD01 model to all observed data (see Mazzei \& Barbaro 2011). For comparison, cols. 7-9 report Sp., $E(B-V)$, and $R_{V}$ values for seven common stars from Wegner (2002).

this group of stars with anomalous $R_{V}$ and normal $\lambda_{\max }$. Detailed modeling for each specific star is beyond the scope of this paper.

\subsection{Numerical modeling and results}

\subsubsection{Numerical method}

Inverse modeling has frequently been used to infer the grain size distribution of dust grains in the ISM of the Milky Way (Kim \& Martin 1995), and in nearby galaxies (e.g., Small Magellanic Cloud (Clayton et al. 2003). Draine \& Fraisse (2009) used the Levenberg-Marquart (LM) method to infer both the grain size distribution and alignment function of interstellar grains in the Galaxy characterized by the typical values of $R_{V}=3.1$ and $\lambda_{\max }=0.55 \mu \mathrm{m}$. A simulation-based inversion technique was developed in Hoang et al. $(2013,2014)$ to find the best-fit grain size distribution and alignment function for interstellar grains in the SNe Ia hosted galaxies with anomalous extinction and polarization data. Although the Monte Carlo simulations demonstrate some advantage (e.g., problem with local minima), its convergence is much slower than the LM method. Thus we adopted the LM method for our modeling.

The goodness of fit of the model $F_{\text {mod }}$ to observed data $F_{\text {obs }}$ is governed by $\chi_{F}^{2}$, which is defined as follows:

$\chi_{F}^{2}=\sum_{i} \frac{\left(F_{\mathrm{mod}}\left(\lambda_{i}\right)-F_{\mathrm{obs}}\left(\lambda_{i}\right)\right)^{2}}{F_{\mathrm{err}}\left(\lambda_{i}\right)^{2}}$,

where $F_{\text {err }}(\lambda)$ is the error in the measurement at wavelength $\lambda$.

When we assume the same errors at all wavelengths, the total $\chi^{2}$ can be written as

$\chi^{2}=\chi_{\mathrm{ext}}^{2}+\eta_{\mathrm{pol}} \chi_{\mathrm{pol}}^{2}+\chi_{\mathrm{vol}}^{2}$

where $\chi_{\text {ext }}^{2}$ and $\chi_{\text {pol }}^{2}$ are evaluated using Eq. (17) for $F=A$ and $F=P$, respectively, $\chi_{\text {vol }}^{2}$ describes the volume constraint determined by the depletion of elements into dust, and $\eta_{\text {pol }}$ is a coefficient introduced to adjust the fit to the polarization. The initial value of $\eta_{\text {pol }}=1$ is chosen. When the fit to the polarization is poor, we can increase $\eta_{\text {pol }}$. Here, we evaluate $\chi_{\mathrm{vol}}^{2}=\chi_{\mathrm{vol}, \mathrm{sil}}^{2}+\chi_{\mathrm{vol}, \mathrm{carb}}^{2}=\left(V_{\mathrm{sil}} / V_{\mathrm{sil}, 0}-1\right)^{2}+\left(V_{\text {carb }} / V_{\text {carb }, 0}-1\right)^{2}$, where $V_{\text {sil,0 }}=2.98 \times 10^{-27} \mathrm{~cm}^{3}$ per $\mathrm{H}$ nucleon and $V_{\text {carb }, 0}=$ $2.07 \times 10^{-27} \mathrm{~cm}^{3}$ per $\mathrm{H}$ nucleon (see WD01).

We search the best-fit values of $\alpha_{j}, \beta_{j}, a_{t, j}, a_{c, j}$, and $c_{j}$, where $j=$ sil, carb and two parameters for grain alignment $\left(a_{\mathrm{ali}}, f_{\mathrm{min}}\right)$ by minimizing $\chi^{2}$ (Eq. (18)) using the LM method from the publicly available package lmfit-py ${ }^{1}$. The errors from observed data are assumed to be $10 \%$.

We note that in WD01, the parameter $a_{c \text {,sil }}$ was fixed to $0.1 \mu \mathrm{m}$. However, Mazzei \& Barbaro (2008) found that the best fit to the extinction for these anomalous stars requires $a_{c \text {,sil }}$ to be reduced to $0.01 \mu \mathrm{m}$, which corresponds to most Si being present in small grains of $a \leq 0.01 \mu \mathrm{m}$. We treated $a_{c, \text { sil }}$ as a model parameter. Furthermore, because we have $R_{V}<4$, grain growth is not expected, thus we constrained the size cutoff parameters $a_{c, \text { carb }} \leq 0.5 \mu \mathrm{m}$ and $a_{c, \text { sil }} \leq 0.5 \mu \mathrm{m}$.

\subsubsection{Model setup}

The sightlines of the considered stars have anomalous extinction curves, with lower $R_{V}$ than the standard value of $R_{V}=3.1$ for the Milky Way. However, the polarization data appear to be normal, with a peak wavelength $\lambda_{\max }>0.4 \mu \mathrm{m}$. For our inverse modeling, we accordingly considered a fixed extinction curve described by a low value of $R_{V}=2.5$. For the polarization data, we considered six different values of $\lambda_{\max }=$ $0.45,0.51,0.53,0.55,0.60$, and $0.65 \mu \mathrm{m}$, which fully covers the range of $\lambda_{\max }$ inferred from observations shown in Table 4. For a given $R_{V}$, we generated (i.e., constructed) the extinction data

1 http://cars9.uchicago.edu/software/python/lmfit/ index.html 

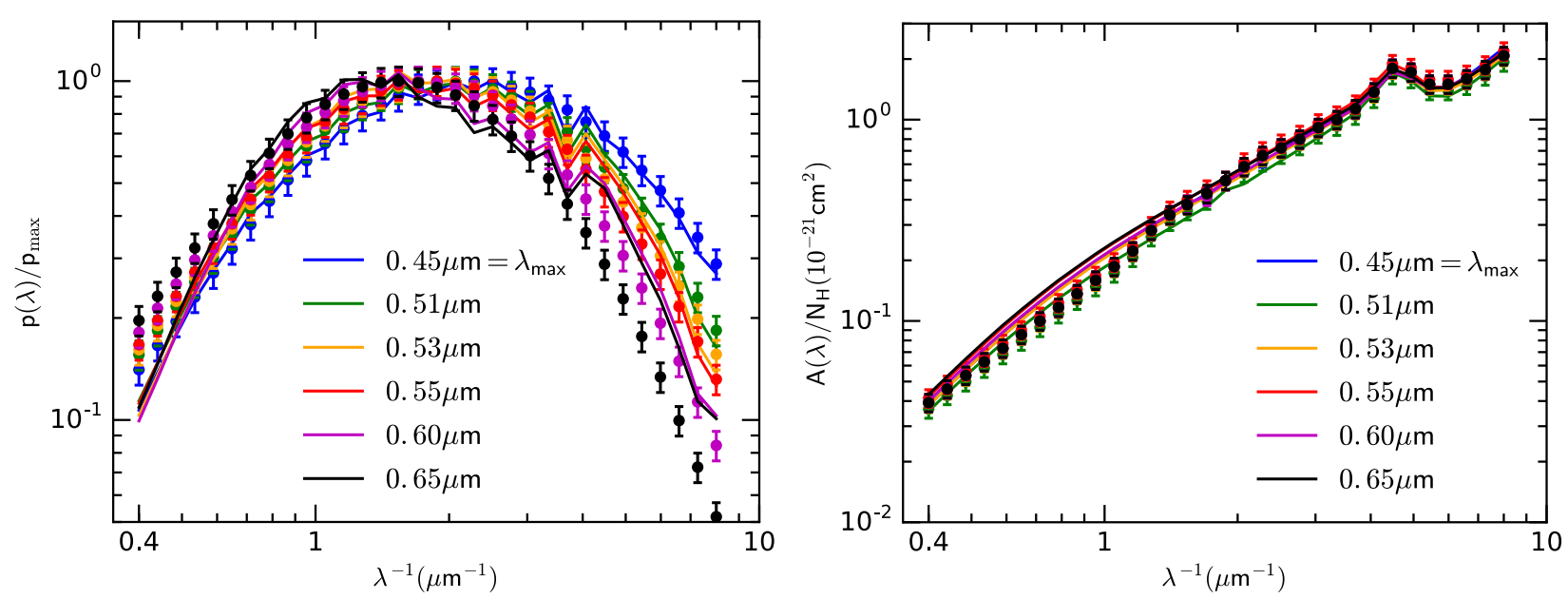

Fig. 5. Left panel: best-fit models vs. generated (i.e., constructed) polarization curves for six different models described by $\lambda_{\max }$. Right panel: best-fit models vs. generated extinction curves with a low $R_{V}=2.5$. Filled circles show the generated data, and solid lines show our best-fit models.

(hereafter, generated extinction curves) using the Cardelli et al. (1989) extinction law. For a given $\lambda_{\max }$, we generated the polarization data (hereafter, generated polarization curves) using the Serkowski curve with $K=k_{1} \lambda_{\max }+k_{2}$ (see Hoang 2017 for details). Here, we adopted a standard relationship with $k_{1}=1.66$ and $k_{2}=0.01$ from Whittet et al. (1992).

Because the extinction and polarization data in the far-UV $(\lambda<0.25 \mu \mathrm{m})$ toward the considered stars are unavailable, we did not attempt to invert the data in the far-UV, which is mainly contributed by ultrasmall grains (including PAHs). Thus, we considered $\lambda=0.25-2.5 \mu \mathrm{m}$ and computed the extinction and polarization model given by Eqs. (15) and (16), respectively. We used 32 bins of grain size in the range from $a_{\text {min }}=3.5 \AA$ to $a_{\max }=1 \mu \mathrm{m}$ and 32 wavelength bins.

Furthermore, we note that while we used the standard Serkowski curve to generate the polarization data, observational studies show differences in the amount of UV polarization relative to that in the visual Serkowski curve. Clayton et al. (1995) found that UV polarimetry measurements of 7 out of 14 sightlines with $\lambda_{\max } \geq 0.54 \mu \mathrm{m}$ agree well with an extrapolation of the Serkowski curve into the UV, while the other 7 sightlines with $\lambda_{\max } \leq 0.53 \mu \mathrm{m}$ show polarization excess compared to the Serkowski extrapolation. They found a relationship between $\lambda_{\max }^{-1}$ and the relative UV polarization $p\left(6 \mu \mathrm{m}^{-1}\right) / p_{\max }$ (see also Martin et al. 1999). Anderson et al. (1996) found that at least half of their sample of 35 sightlines for which they have reliable UV observations did not agree well compared to the Serkowski extrapolation from visual and near-IR parameters. An increase/decrease in the UV polarization would lead to an increase/decrease in the degree of alignment of small grains inferred from simulations, whereas the alignment of large grains $(a>0.1 \mu \mathrm{m})$ that dominates the visible-IR polarization would be unchanged. The grain size distributions would be slightly changed (see Hoang et al. 2014).

The important constraint for the polarization model (see Sect. 6.2.2) and the alignment function $f_{\text {ali }}(a)$ is that for the maximum polarization efficiency $p_{\max } / A\left(\lambda_{\max }\right)=3 \% \mathrm{mag}^{-1}$ (see Draine 2003 for a review), we expect that the conditions for grain alignment are optimal, which corresponds to the case in which the alignment of large grains can be perfect, and the magnetic field is regular and perpendicular to the line of sight. Thus, we set $f_{\text {ali }}\left(a=a_{\max }\right)=1$.

\subsubsection{Results}

Figure 5 shows the best-fit polarization and extinction curves for the different $\lambda_{\max }$. The fit to the extinction curve is good, but the model overestimates the extinction for $\lambda \geq 1 \mu \mathrm{m}$ for $\lambda_{\max }=0.53-0.65 \mu \mathrm{m}$. For the polarization, the fit is excellent for $\lambda_{\max }<0.6 \mu \mathrm{m}$, but the model (see Sect. 6.2.2) overestimates the polarization at $\lambda<0.25 \mu \mathrm{m}$ for $\lambda_{\max }=0.6 \mu \mathrm{m}$ and $0.65 \mu \mathrm{m}$.

Figure 6 shows the best-fit size distributions for silicate and carbonaceous grains. The size distribution appears to change slightly with $\lambda_{\max }$, which is expected due to the fixed $R_{V}$. To reproduce the typical $\lambda_{\max }$, there must be a population of large silicate grains of $a \geq 0.1 \mu \mathrm{m}$. This is different from the results obtained by Mazzei \& Barbaro (2008), where the authors only performed the fitting to the extinction curves and found a lack of large silicate grains, but large grains in the carbonacous grain size distribution.

Figure 7 shows the best-fit alignment function for the different $\lambda_{\max }$. When $\lambda_{\max }$ decreases, the alignment function tends to shift to smaller sizes. Moreover, the alignment of small grains $(a<0.05 \mu \mathrm{m})$ is increased with decreasing $\lambda_{\max }$. This trend is consistent with the results from Hoang et al. (2014), where the modeling was done for the cases with normal extinction curves (i.e., $R_{V} \sim 3.1$ ) and excess UV polarization.

\section{Discussion}

\subsection{Comparison to supernovae la and normal Galactic stars}

The main aim of this work is to investigate the polarization profiles of Galactic stars with low $R_{V}$ values, with the aim to try to find similar polarization behavior, as we observe in highly reddened SNe Ia with low $R_{V}$ values, with the polarization degree rising toward blue wavelengths (see, e.g., Fig. 2 in Patat et al. 2015). However, none of the stars with anomalous extinction sightlines in our sample display such polarization curves that steeply rise toward the blue (Fig. 1).

Figure 2 shows our sample of stars with anomalous extinction sightlines in the $\lambda_{\max }-K$ plane compared to a sample of SNe Ia from Patat et al. (2015) and Zelaya et al. (2017; see Appendix B). Despite the low $R_{V}$ values, our sample has normal polarization curves with a mean $\lambda_{\max } \sim 0.53 \mu \mathrm{m}$. The Serkowski parameters $K$ and $\lambda_{\max }$ are related $(\rho=0.87$, 

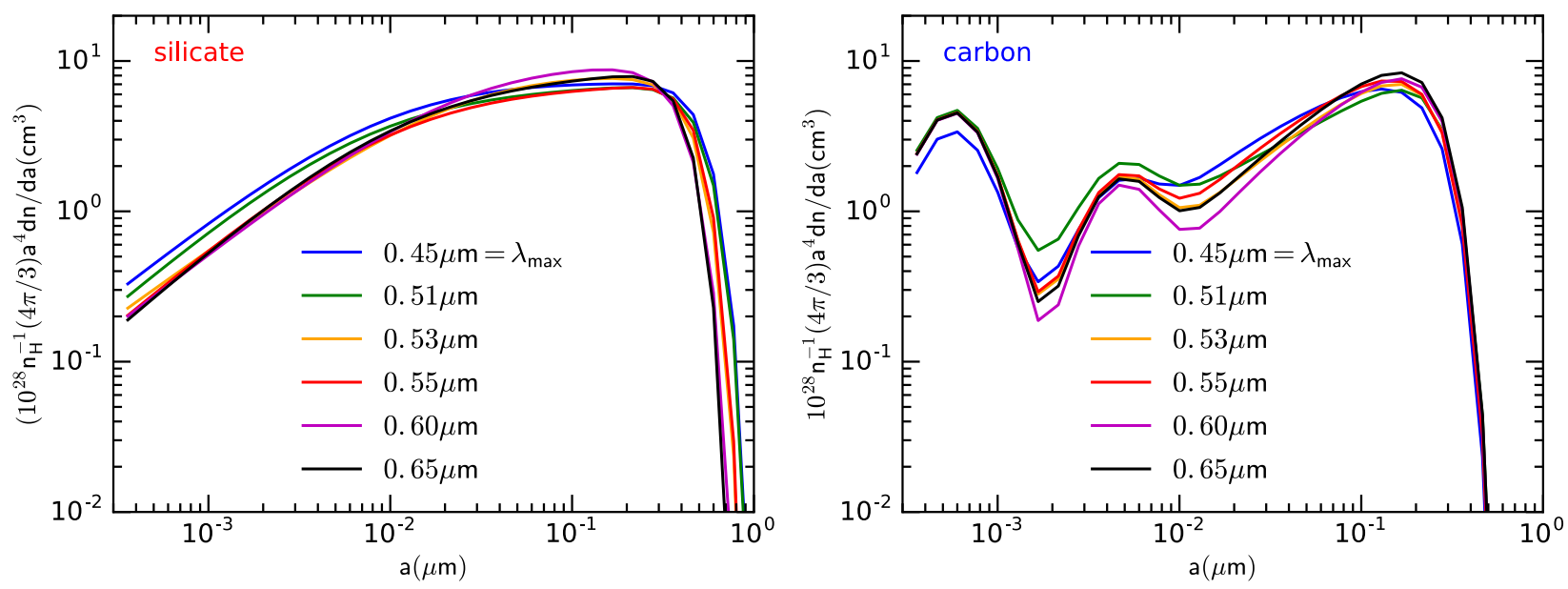

Fig. 6. Best-fit grain size distribution for silicate (left panel) and carbonaceous grains (right panel). Six different models described by $\lambda_{\max }$ are considered. Large silicate grains of size $a \geq 0.1 \mu \mathrm{m}$ are present to reproduce normal $\lambda_{\max }$.

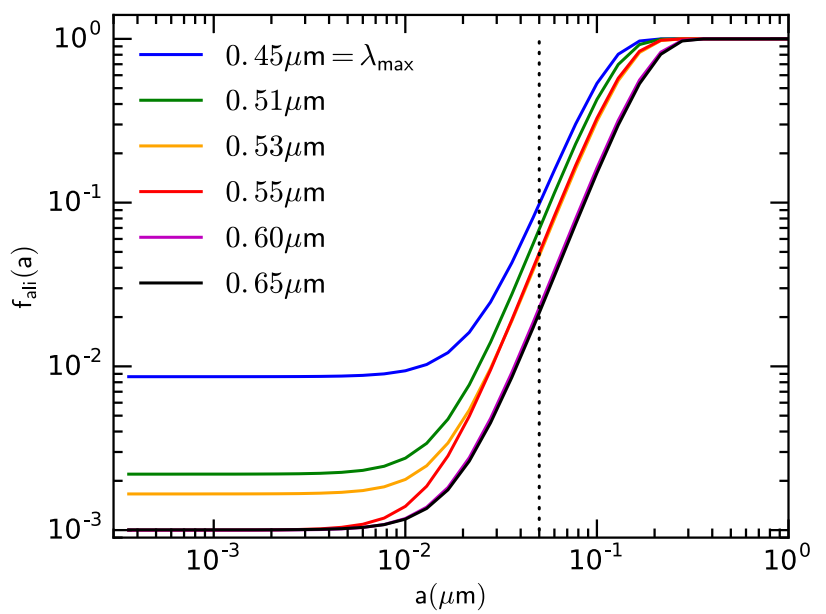

Fig. 7. Best-fit alignment function of silicates for the different models given by $\lambda_{\max }$. The dotted line marks the typical grain size $a=0.05 \mu \mathrm{m}$. The alignment function tends to shift to smaller sizes as $\lambda_{\max }$ decreases.

$p=3 \times 10^{-5}$ ), and can be described as a linear function of $\lambda_{\max }$ : $K=1.13 \pm 0.34+(4.05 \pm 0.64) \lambda_{\max }$. This is steeper than the empirical relationship found by Whittet et al. (1992): $K=0.01 \pm$ $0.05+(1.66 \pm 0.09) \lambda_{\max }$ (see also Wilking et al. 1980, 1982). However, the $K-\lambda_{\max }$ relationship in Whittet et al. (1992) was determined from a chosen sample of sightlines toward stars with a variety of interstellar environments, including dense clouds, diffuse clouds, and low-density interstellar material.

Figure 3 shows a direct comparison of our sample with the Whittet et al. (1992) sample and the Large Interstellar Polarization Survey (LIPS) sample (Bagnulo et al. 2017). Despite the difference in the slope, our sample is consistent within $3 \sigma$ with the Whittet et al. (1992) sample and also coincides well with the LIPS sample, which has many outliers from the $K-\lambda_{\max }$ relationship.

For comparison, $\mathrm{SNe}$ Ia with low $R_{V}$ values have $\lambda_{\max } \lesssim 0.45 \mu \mathrm{m}$, and higher $K$ values, above the Whittet et al. (1992) $\lambda_{\max }-K$ relationship, due to the steep rise of the polarization curve toward the blue. There are two exceptions: SN $2002 \mathrm{fk}$ and SN 2007af, which are consistent (within the errors) with the Galactic stars sample and have $\lambda_{\max }$ of $\sim 0.44 \mu \mathrm{m}$ and $\sim 0.74 \mu \mathrm{m}$, respectively (Table B.1).
Cikota et al. (2017b) noted that some post-AGB stars (protoplanetary nebula, PPN) have polarization curves rising toward the blue, which are produced by CSM scattering (Oppenheimer et al. 2005). These polarization curves are remarkably similar to those observed toward highly reddened SNe Ia. They suggested that these polarization curves observed toward highly reddened $\mathrm{SNe}$ Ia might also be produced by CSM dust scattering. Furthermore, these SNe Ia might explode within a PPN. The main caveat is that if the polarization is produced by scattering, the polarization angles, which carry the geometrical imprint of the dust distribution in the PPN, are expected to be randomly orientated, while the observed polarization angles in sightlines of highly reddened SNe Ia show an alignment with the structure of their host galaxies, probably as a consequence of dust-grain alignment along the local magnetic field (Patat et al. 2015, see also Hoang 2017).

\section{2. $R_{V}-\lambda_{\max }$ relationship}

Serkowski et al. (1975) found that $\lambda_{\max }$ is correlated with the ratios of color excess, for example, $E(V-K) / E(B-V)$, and thus to the total-to-selective extinction ratio $R_{V}$. They found that $R_{V}=5.5 \lambda_{\max }$, where $\lambda_{\max }$ is in $\mu \mathrm{m}$. Whittet \& van Breda (1978) deduced $R_{V}=(5.6 \pm 0.3) \lambda_{\max }$ using a sample of carefully selected normal stars and therewith confirmed the result by Serkowski et al. (1975). Clayton \& Mathis (1988) confirmed that the $\lambda_{\max }-R_{V}$ relationship is real, and derived $R_{V}=(-0.29 \pm$ $0.74)+(6.67 \pm 1.17) \lambda_{\max }$ using a modified extinction law in which they forced the extinction to zero at infinite wavelengths. They also concluded that the variations in $\lambda_{\max }$ are produced by the dust grains' size distribution and not by a variation in the alignment of the dust grains.

However, our sample of anomalous extinction sightlines does not show any significant correlation between $\lambda_{\max }$ and $R_{V}$. The correlation coefficient is $\rho \leq 0.26$. The $\lambda_{\max }$ values are higher than expected from the $\lambda_{\max }-R_{V}$ relationship given in Whittet $\&$ van Breda (1978), for instance.

The most likely explanation is that while not all dust types contribute to polarization, all dust types do contribute to extinction, and thus to the $R_{V}$ value. The polarization curve mainly depends on the dust grain size distribution of silicates because magnetic alignment is more efficient for silicates than, for instance, for carbonaceous dust grains (Somerville et al. 1994). 
It has been shown in previous works that there is not necessarily a correlation between $R_{V}$ and $\lambda_{\max }$. Whittet et al. (1994) measured linear polarization toward the Chamaeleon I dark cloud and found only a weak correlation between $R_{V}$ and $\lambda_{\max }$. Whittet et al. (2001) presented observations of interstellar polarization for stars in the Taurus dark cloud and found no clear trend of increasing $R_{V}$ with $\lambda_{\max }$ (see their Fig. 9). Their sample shows normal optical properties, with $R_{V} \sim 3$, while the $\lambda_{\max }$ values are higher than expected from observations toward normal stars (e.g., Whittet \& van Breda 1978). They suggested that the poor $R_{V}-\lambda_{\text {max }}$ correlation can be explained by dust grain size dependent variations in alignment capabilities of the dust grains. The LIPS data (Bagnulo et al. 2017) do not follow any $R_{V}-\lambda_{\max }$ relationship either.

Another possibility is that the $R_{V}$ values presented in Mazzei \& Barbaro (2011) are lower than the true values. The CCM $R_{V}$ values (listed in Table 4 ) were determined by bestfitting the IR observations with the CCM law (Table 1 in Mazzei \& Barbaro 2011), and are consistent with estimates of $R_{V}$ values following the methods in Fitzpatrick (1999). The $R_{V}$ values in Table 4 (taken from Table 4 in Mazzei \& Barbaro 2011) were determined by best-fitting the whole extinction curve with the WD01 model (see Mazzei \& Barbaro 2011). It is important to note that Fitzpatrick \& Massa (2007) showed that the relations between $R_{V}$ and UV extinction can arise from sample selection and method, and that there is generally no correlation between the UV and IR portions of the Galactic extinction curves.

Wegner (2002) presented 436 extinction curves covering a wavelength range from UV to near-IR, including seven stars from our subsample: HD 14357, HD 37061, HD 54439, HD 78785, HD 96042, HD 152245, and HD 226868. They determined the $R_{V}$ values by extrapolating the ratio $E(\lambda-V) / E(B-V)$ to $1 / \lambda=0$, where the extinction should be zero, and found slightly higher values. The $E(B-V)$ and $R_{V}$ values determined in Wegner (2002) of seven common stars are listed in Table 4. The $R_{V}$ values determined by Wegner (2002) are $1.4 \pm 0.2$ times higher than the $R_{V}$ values determined in Mazzei \& Barbaro (2011) by best-fitting the WD01 model to observations, and $1.2 \pm 0.2$ times higher than the CCM $R_{V}$ values determined by best-fitting the IR observations with the CCM law. A caveat of the extrapolation method is that IR emission from possible CS shells around Be stars might suggest increased $R_{V}$ values (Wegner 2002).

We also note that the $E(B-V)$ values used in Mazzei \& Barbaro (2011, and originally taken from Savage et al. 1985) of the common stars are $\sim 1.1 \pm 0.1$ times higher than those in Wegner (2002), which also contributes to lower $R_{V}$ values in Mazzei \& Barbaro (2011) compared to values in Wegner (2002), and that the spectral types used in Savage et al. (1985, used in Mazzei \& Barbaro 2011) are different than those in Wegner (2002 Table 4). Wegner (2002) took the spectral classification from the SIMBAD database and compared the most recent estimate with the most frequent estimate. The author found that for about $60 \%$ of his sample, the most frequent and most recent spectral and luminosity classes are the same, while there is a difference of 0.05 in the spectral class for about $18 \%$ of stars, of 0.1 for $16 \%$, and of 0.2 spectral class for $6 \%$. This shows that the extinction curves of the same targets, computed by different authors, are slightly different.

Figure 8 shows the $R_{V}$ values determined by best-fitting the IR extinction curves with the CCM law as a function of $\lambda_{\max }$ compared to the values determined from the best fit of the observed extinction curves by the WD01 models (both taken from Mazzei \& Barbaro 2011) and the $R_{V}$ values for seven

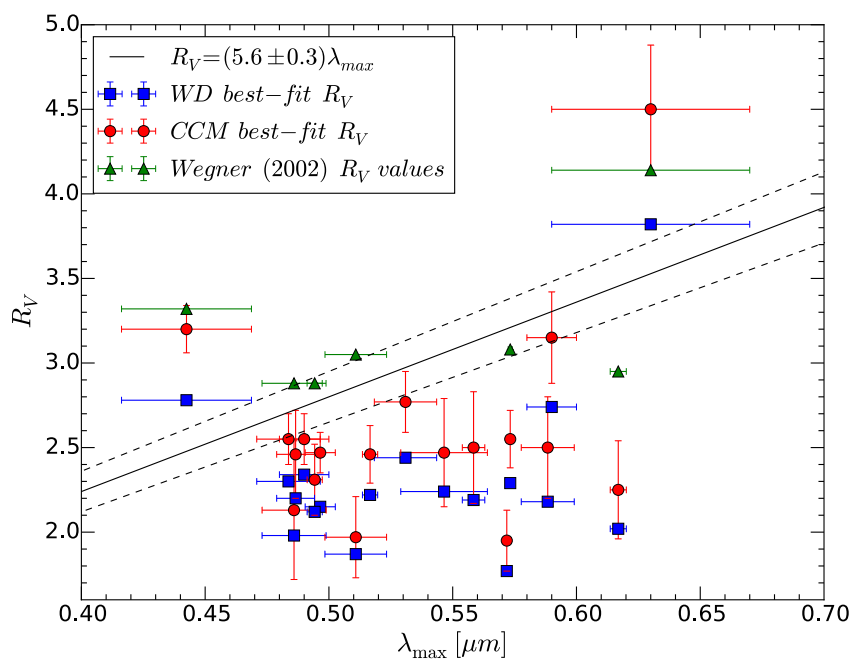

Fig. 8. $R_{V}-\lambda_{\max }$ plane. The red dots mark the CCM $R_{V}$ values as a function of $\lambda_{\max }$ determined by best-fitting the IR extinction curves with the CCM extinction law, the blue dots mark the $R_{V}$ values determined by fitting the WD01 model to all observations, and the green triangles mark the $R_{V}$ values from Wegner (2002) determined by the extrapolation method (see Table 2). The black line shows the $R_{V}$ relationship from Whittet \& van Breda (1978) and its $1 \sigma$ uncertainty.

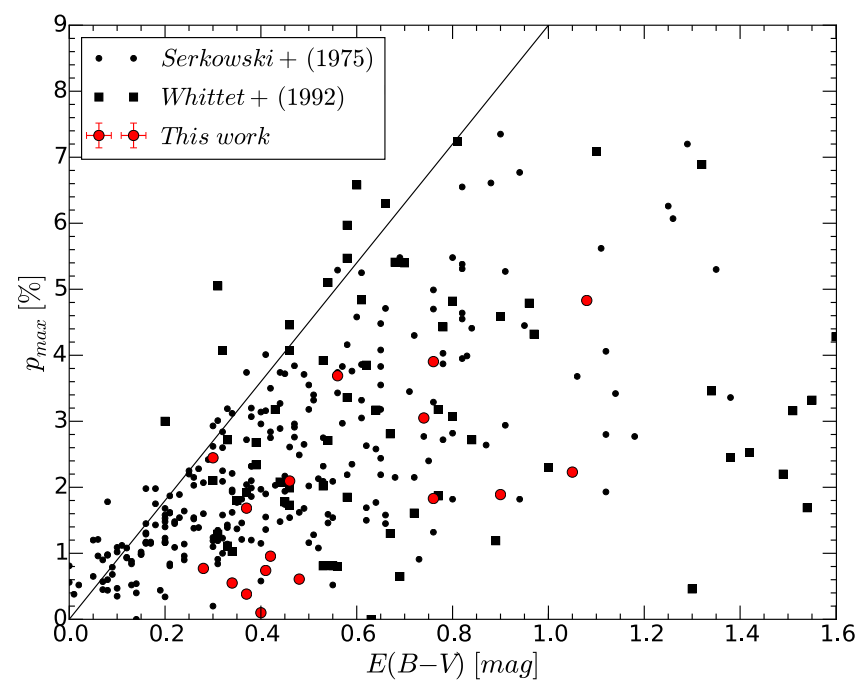

Fig. 9. Maximum interstellar polarization $p_{\max }$ vs. color excess $E(B-V)$ of stars from Whittet et al. (1992 black squares), Serkowski et al. (1975 black circles from), compared to our observed sample (red circles). The straight line denotes the upper limit $p_{\max }(\%)=9.0 E(B-V)$ mag defined by Serkowski et al. (1975).

common stars from Wegner (2002), determined by the extrapolation method (see Table 2). Four of seven stars with $R_{V}$ values determined by Wegner (2002) lie within the Whittet \& van Breda (1978) $R_{V}$ relationship, while most of the stars with $R_{V}$ values determined by best-fitting the CCM law and the WD01 model (Mazzei \& Barbaro 2011) are below the Whittet \& van Breda (1978) relationship.

\section{3. $p_{\max }-E(B-V)$ relationship}

There is no clear correlation between the maximum polarization and color excess. Figure 9 shows the Serkowski et al. (1975) and Whittet et al. (1992) sample in the $p_{\max }-E(B-V)$ plane compared to our sample of stars with anomalous sightlines. 

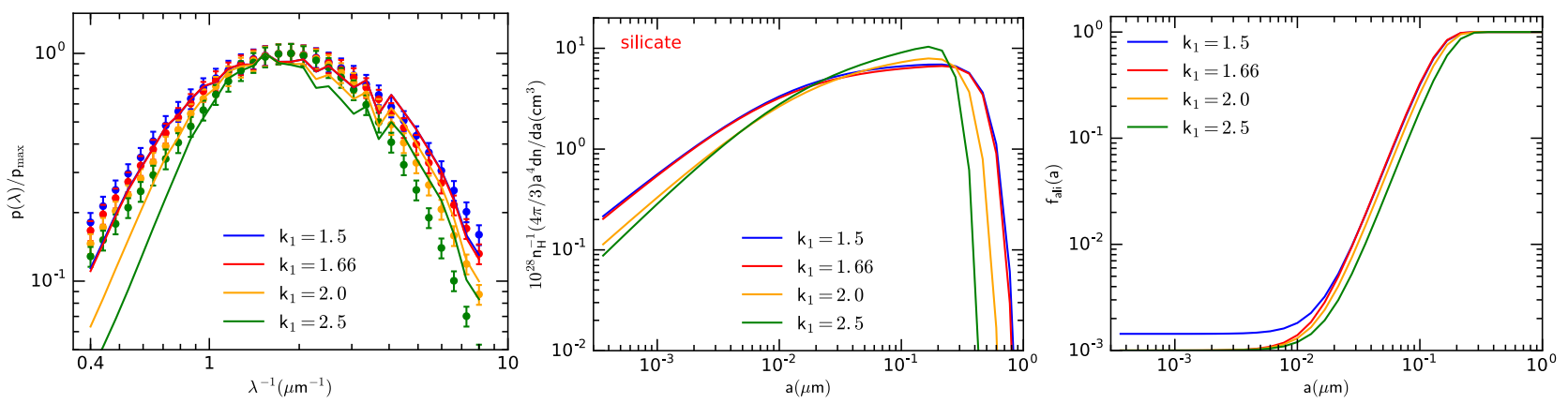

Fig. 10. Polarization curves (left panel), best-fit size distribution (middle panel), and best-fit alignment function (right panel) for the different values of $k_{1}$, where $K=k_{1} \lambda_{\max }+0.01$. The value of $\lambda_{\max }=0.55 \mu \mathrm{m}$ is fixed.

The scattered data in the plot shows no dependence of maximum polarization on reddening, but there is an upper limit depending on reddening (Serkowski et al. 1975) that is rarely exceeded: $p_{\max }(\%)=9.0 E(B-V)$ mag. We calculated the mean of the ratio $\left\langle p_{\max } / E(B-V)\right\rangle$ for the different samples: $\left\langle p_{\max } / E(B-V)\right\rangle=6.2 \pm 3.8 \% \mathrm{mag}^{-1}$ for the Serkowski et al. (1975) sample, $\left\langle p_{\max } / E(B-V)\right\rangle=4.6 \pm 3.4 \% \mathrm{mag}^{-1}$ for the Whittet et al. (1992) sample, and $\left\langle p_{\max } / E(B-V)\right\rangle=$ $3.3 \pm 2.1 \% \mathrm{mag}^{-1}$ for our sample. The low $\left\langle p_{\max } / E(B-V)\right\rangle$ ratio of our sample might also indicate that the silicate dust grains do not align as efficiently as the Serkowski et al. (1975) and Whittet et al. (1992) samples, but because of the small number of stars in our sample, we cannot draw such conclusions with high certainty. Another possible reason for the low ratios of $p_{\text {max }} / E(B-V)$ is a small angle between the direction of the magnetic field and the line of sight, that is expected to reduce $p_{\max } / E(B-V)$, as discussed in previous works, for example, Hoang et al. (2014).

\subsection{Which dust properties determine $\lambda_{\max }$ ?}

For a given grain shape and dust optical constant, Eq. (16) reveals that the polarization spectrum is determined by $d n / d a \times f_{\text {ali }}$, which is considered the size distribution of aligned grains, while the extinction (i.e., $R_{V}$ ) in Eq. (15) is only determined by $d n / d a$. Thus, both a change in $d n / d a$ and $f_{\text {ali }}$ affect the polarization spectrum.

Our simultaneous fitting to the extinction and polarization demonstrate that both grain alignment and size distribution are required to change in order to reproduce the variation of $\lambda_{\max }$ (see Figs. 6 and 7). However, the change in grain alignment is more prominent. Figure 7 shows that the alignment of small grains required to reproduce $\lambda_{\max }=0.45 \mu \mathrm{m}$ is an order of magnitude higher than that required for $\lambda_{\max }=0.55 \mu \mathrm{m}$. We note that the modeling here is carried out for a constant $R_{V}$. In the lines of sight where grain growth can take place, resulting in the increase of $R_{V}$, we expect both grain evolution and alignment to contribute to the variation of $\lambda_{\max }$ and $K$.

To test whether grain evolution can reproduce the observed data, we reran our simulations for the same six models by fixing the alignment function that reproduces the "standard" polarization curve with typical value $\lambda_{\max }=0.55 \mu \mathrm{m}$. The size distributions $d n_{j} / d a$ was varied. We found that the variation of $d n / d a$ can reproduce the observed data to a satisfactory level only for the cases of $\lambda_{\max }=0.51-0.55 \mu \mathrm{m}$, that is, $\lambda_{\max }$ is not much different from the standard value. Meanwhile, the fit to the models is poor when $\lambda_{\max }$ differs much from the typical value of $0.55 \mu \mathrm{m}$. This indicates that grain evolution alone cannot explain the wide range of $\lambda_{\max }$ that is observed.

\subsection{Why is $K$ correlated to $\lambda_{\max }$ ?}

The dependence of $K$ on $\lambda_{\max }$ appears to be an intrinsic property of the polarization. The Serkowski curve shows that a smaller $K$ corresponds to a broader polarization profile. From the inverse modeling for a constant $R_{V}$, we find that the grain alignment function becomes broader (narrower) for lower (higher) values of $\lambda_{\max }$ as well as of $K$. This feature can be explained as follows. Each aligned grain of size $a$ produces an individual polarization profile $C_{\text {pol }}$ with the peak at $\lambda \sim 2 \pi a$ (see Fig. 1 in Hoang et al. 2013). The polarization spectrum is the superimposition/integration over all grain sizes that are aligned. When the alignment function is broader, the superposition produces a broader polarization profile, or smaller $K$.

\subsection{Deviation of $K$ from the standard value}

To explore the dust properties underlying the deviation of $K$ from the typical value, we performed the fit for a fixed $\lambda_{\max }$ and varying $k_{1}$ (in $K=k_{1} \lambda_{\max }+0.01$ ).

Figure 10 shows the best-fit models (left panel), alignment function (right panel), and size distribution (middle panel) for a fixed $\lambda_{\max }=0.55 \mu \mathrm{m}$ and varying $K$. We find that the increase of $K$ is produced by the decrease of large $\mathrm{Si}$ grains $(a>0.2 \mu \mathrm{m})$. At the same time, the alignment is shifted to the larger size when $K$ increases, which is shown in Fig. 7 when $\lambda_{\max }$ (and $K$ ) is increased.

\subsection{Relationship between $R_{\mathrm{Si}}$ and $\lambda_{\max }$}

Figure 11 shows the dependence of $R_{\mathrm{Si}}$ on $\lambda_{\max }$. There is a slight decrease of $R_{\mathrm{Si}}$ with increasing $\lambda_{\max }$. This is straightforward because small grains are required to reproduce higher polarization in the UV when $\lambda_{\max }$ decreases.

The values $R_{\mathrm{Si}}$ from Fig. 4 were inferred from fitting only the extinction data (Mazzei \& Barbaro 2011). Thus, there is no direct relation between such inferred $R_{\mathrm{Si}}$ and $\lambda_{\max }$ that describes the polarization curve. It is known that a dust model that fits the extinction well may not reproduce the polarization data (e.g., $\mathrm{dn} / \mathrm{da} \sim \mathrm{a}^{-3.5}$ law by Mathis et al. 1977; see, e.g., Kim \& Martin 1995; Draine \& Fraisse 2009).

The scale difference between $R_{\mathrm{Si}}$ shown in Figs. 4 and 11 arises from the different ways of modeling. In Mazzei \& Barbaro (2011), to reproduce the extinction curve with low $R_{V}$ values, dust grains were found to be rather small $(\lessgtr 0.05 \mu \mathrm{m})$, leading to a high value of $R_{\mathrm{Si}}$. Such a dust model cannot reproduce the polarization data with standard $\lambda_{\max }$ that requires aligned large grains (i.e., larger $0.1 \mu \mathrm{m}$ ). Our best-fit models that simultaneously fit 


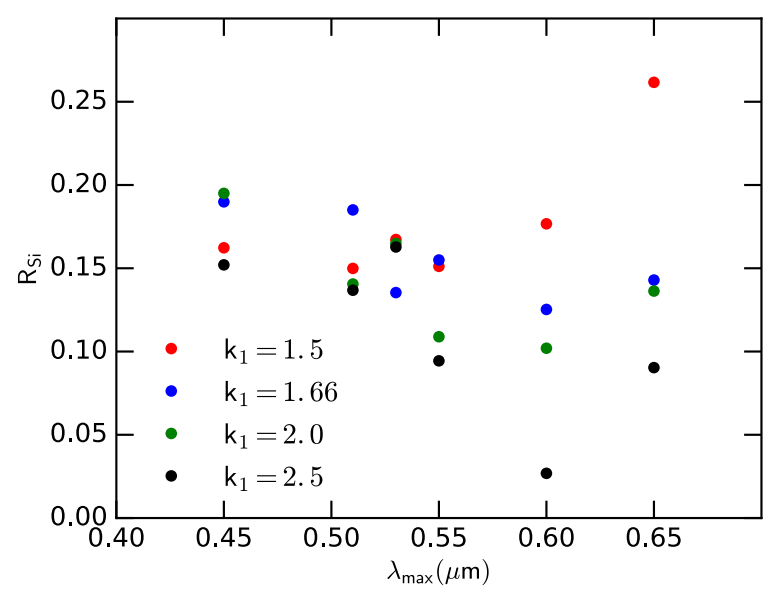

Fig. 11. Relative ratio of Si abundance in very small and large grain sizes vs. $\lambda_{\max }$.

extinction and polarization data provide different size distributions that have large grains, leading to smaller $R_{\mathrm{Si}}$.

\section{Summary and conclusions}

We investigated the linear polarization of 17 sightlines to Galactic stars with anomalous extinction laws and low total-toselective visual extinction ratio, $R_{V}$, selected from the Mazzei \& Barbaro (2011) sample, and adopted a simple dust model that can reproduce the observed sightlines with low $R_{V}$ values and normal polarization curves. To do this, we adopted the Weingartner \& Draine (2001) dust model and a picket-fence alignment model to compute extinction and polarization curves (see Sect. 6.2.2). Our results are summarized below.

1. Galactic stars with anomalous extinction sightlines, with low $R_{V}$ values, show "normal" polarization curves with a mean $\lambda_{\max } \sim 0.53 \mu \mathrm{m}$. This can be explained by considering that e not all dust that contributes to extinction also contributes to polarization. The polarization mainly depends on the dust grain size distribution of silicates, because grain alignment is more efficient for silicates than, for instance, for carbonaceous dust grains (Somerville et al. 1994), whereas $R_{V}$ is strongly dependent on carbonaceous grains too.

2. There is no significant $R_{V}-\lambda_{\max }$ relation in our sample (Fig. 8). The $\lambda_{\max }$ values in our sample are higher than compared to normal stars that follow the empirical $R_{V}-\lambda_{\max }$ relationship described by Whittet \& van Breda (1978), for instance.

3. Despite the low $R_{V}$ value, there is no similarity between the polarization curves in the investigated sample and the polarization curves observed in reddened SNe Ia with low $R_{V}$ values. The polarization curves are consistent with a sample of Galactic stars observed by Whittet et al. (1992) within $3 \sigma$ (Fig. 2).

4. The Serkowski parameters $K$ and $\lambda_{\max }$ are correlated. However, we find a steeper slope $(K=-1.13 \pm 0.34+(4.05 \pm$ $0.64) \lambda_{\max }$ ) in our sample than in the empirical relationship found by Whittet et al. (1992), for example.

5. Simulations show that to reproduce a polarization curve with the normal $\lambda_{\max }$ and low $R_{V}$, there must be a population of large interstellar silicate grains of size $a \geq 0.1 \mu \mathrm{m}$. This is different compared to results by Mazzei \& Barbaro (2008); Mazzei \& Barbaro (2011), who only best-fit the extinction curves and found a lack of such large Si grains, but did find large carbonaceous grains. Moreover, variations in grain alignment and size distribution together are required to reproduce the variation in $\lambda_{\max }$ for a fixed, low, $R_{V}$ value. However, a change in grain alignment has a greater effect.

6. By comparing the $R_{V}$ values of the sample here considered with those in Wegner (2002) for a subset of our stars, we find some differences that are probably due to a different spectral classification and/or luminosity class adopted to derive their extinction curves (see Sect. 7.2). The $\lambda_{\max }$ value that we measure and the deviation from the empirical $R_{V}-\lambda_{\max }$ relationship may also suggest a spectral misclassification of some stars by Savage et al. (1985).

7. The $K-\lambda_{\max }$ relation appears to be an intrinsic property of the polarization. Simulations show that for a fixed $R_{V}$, the grain alignment function becomes narrower (broader) for a lower (higher) value of $\lambda_{\max }$ and $K$ (see Sect. 7.5).

8. An increase in Serkowski parameter $K$ and deviation from the standard value in the $K-\lambda_{\max }$ plane can be reproduced by a decreasing contribution of large Si grains (Fig. 10).

Acknowledgements. We thank the anonymous referee for useful comments that significantly improved the paper. This work is partially based on observations collected at the German-Spanish Astronomical Center, Calar Alto, jointly operated by the Max-Planck-Institut für Astronomie Heidelberg and the Instituto de Astrofisica de Andalucia (CSIC). T.H. acknowledges the support from the Basic Science Research Program through the National Research Foundation of Korea (NRF), funded by the Ministry of Education (2017R1D1A1B03035359). This work is based on observations made with ESO Telescopes at the Paranal Observatory under Program ID 094.C-0686, and partially based on observations collected with the Copernico telescope (Asiago, Italy) of the INAF Osservatorio Astronomico di Padova. We also thank P. Ochner for taking some observations with AFOSC in service time. Some of the data presented in this paper were obtained from the Mikulski Archive for Space Telescopes (MAST). STScI is operated by the Association of Universities for Research in Astronomy, Inc., under NASA contract NAS5-26555. Support for MAST for non-HST data is provided by the NASA Office of Space Science via grant NNX09AF08G and by other grants and contracts. ST acknowledges support from TRR33 "The Dark Universe" of the German Research Foundation.

\section{References}

Altavilla, G., Fiorentino, G., Marconi, M., et al. 2004, MNRAS, 349, 1344 Anderson, C. M., Weitenbeck, A. J., Code, A. D., et al. 1996, AJ, 112, 2726 Andersson, B.-G., Lazarian, A., \& Vaillancourt, J. E. 2015, ARA\&A, 53, 501 Bagnulo, S., Cox, N. L. J., Cikota, A., et al. 2017, A\&A, 608, A146 Cardelli, J. A., Clayton, G. C., \& Mathis, J. S. 1989, ApJ, 345, 245 Chiar, J. E., Adamson, A. J., Whittet, D. C. B., et al. 2006, ApJ, 651, 268 Cikota, A., Deustua, S., \& Marleau, F. 2016, ApJ, 819, 152

Cikota, A., Patat, F., Cikota, S., \& Faran, T. 2017a, MNRAS, 464, 4146 Cikota, A., Patat, F., Cikota, S., Spyromilio, J., \& Rau, G. 2017b, MNRAS, 471, 2111

Clayton, G. C., \& Mathis, J. S. 1988, ApJ, 327, 911

Clayton, G. C., Wolff, M. J., Allen, R. G., \& Lupie, O. L. 1995, ApJ, 445, 947 Clayton, G. C., Wolff, M. J., Sofia, U. J., Gordon, K. D., \& Misselt, K. A. 2003, ApJ, 588, 871

Conley, A., Carlberg, R. G., Guy, J., et al. 2007, ApJ, 664, L13

Coyne, G. V., Gehrels, T., \& Serkowski, K. 1974, AJ, 79, 581

Draine, B. T. 2003, ARA\&A, 41, 241

Draine, B. T., \& Allaf-Akbari, K. 2006, ApJ, 652, 1318

Draine, B. T., \& Fraisse, A. A. 2009, ApJ, 696, 1

Draine, B. T., \& Li, A. 2007, ApJ, 657, 810

ESO 2015, FORS2 User Manual, issue 96.0, VLT-MAN-ESO-13100-1543 (European Southern Observatory)

Fitzpatrick, E. L. 1999, PASP, 111, 63

Fitzpatrick, E. L., \& Massa, D. 2007, ApJ, 663, 320

Folatelli, G., Phillips, M. M., Burns, C. R., et al. 2010, AJ, 139, 120

Foley, R. J., Fox, O. D., McCully, C., et al. 2014, MNRAS, 443, 2887

Giridhar, S., \& Arellano Ferro, A. 2005, A\&A, 443, 297

Goobar, A. 2008, ApJ, 686, L103

Hicken, M., Wood-Vasey, W. M., Blondin, S., et al. 2009, ApJ, 700, 1097

Hoang, T. 2017, ApJ, 836, 13

Hoang, T., \& Lazarian, A. 2016, ApJ, 831, 159

Hoang, T., Lazarian, A., \& Martin, P. G. 2013, ApJ, 779, 152

Hoang, T., Lazarian, A., \& Martin, P. G. 2014, ApJ, 790, 6

Hong, S. S., \& Greenberg, J. M. 1980, A\&A, 88, 194 
A. Cikota et al.: Spectropolarimetry of Galactic stars with anomalous extinction sightlines

Hsu, J.-C., \& Breger, M. 1982, ApJ, 262, 732

Johnson, J. J., \& Jones, T. J. 1991, AJ, 101, 1735

Kessler, R., Becker, A. C., Cinabro, D., et al. 2009, ApJS, 185, 32

Kim, S.-H., \& Martin, P. G. 1995, ApJ, 444, 293

Lampeitl, H., Smith, M., Nichol, R. C., et al. 2010, ApJ, 722, 566

Lazarian, A., Andersson, B.-G., \& Hoang, T. 2015, in Polarimetry of stars and planetary systems, eds. L. Kolokolova, J. Hough, \& A.-C. Levasseur-Regourd (Cambridge, UK: Cambridge Univ. Press), 81

Lü, G., Zhu, C., \& Podsiadlowski, P. 2013, ApJ, 768, 193

Mandel, K. S., Narayan, G., \& Kirshner, R. P. 2011, ApJ, 731, 120

Martin, P. G., Clayton, G. C., \& Wolff, M. J. 1999, ApJ, 510, 905

Mathis, J. S., Rumpl, W., \& Nordsieck, K. H. 1977, ApJ, 217, 425

Mazzei, P., \& Barbaro, G. 2008, MNRAS, 390, 706

Mazzei, P., \& Barbaro, G. 2011, A\&A, 527, A34

Nobili, S., \& Goobar, A. 2008, A\&A, 487, 19

Oliva, E. 1997, A\&AS, 123, 589

Oppenheimer, B. D., Bieging, J. H., Schmidt, G. D., et al. 2005, ApJ, 624, 957

Patat, F., \& Romaniello, M. 2006, PASP, 118, 146

Patat, F., \& Taubenberger, S. 2011, A\&A, 529, A57

Patat, F., Maund, J. R., Benetti, S., et al. 2010, A\&A, 510, A108

Patat, F., Taubenberger, S., Cox, N. L. J., et al. 2015, A\&A, 577, A53

Phillips, M. M., Lira, P., Suntzeff, N. B., et al. 1999, AJ, 118, 1766

Phillips, M. M., Simon, J. D., Morrell, N., et al. 2013, ApJ, 779, 38

Pourbaix, D., Tokovinin, A. A., Batten, A. H., et al. 2004, A\&A, 424, 727

Reindl, B., Tammann, G. A., Sandage, A., \& Saha, A. 2005, ApJ, 624, 532

Riess, A. G., Press, W. H., \& Kirshner, R. P. 1996, ApJ, 473, 588
Savage, B. D., Massa, D., Meade, M., \& Wesselius, P. R. 1985, ApJS, 59, 397 Schmidt, G. D., Elston, R., \& Lupie, O. L. 1992, AJ, 104, 1563

Serkowski, K., Mathewson, D. S., \& Ford, V. L. 1975, ApJ, 196, 261

Somerville, W. B., Allen, R. G., Carnochan, D. J., et al. 1994, ApJ, 427, L47

Trujillo-Bueno, J., Moreno-Insertis, F., \& Sanchez Martinez F. 2002, Astrophysical Spectropolarimetry (Cambridge, UK: Cambridge University Press), 368

Voshchinnikov, N. V. 2012, J. Quant. Spectr. Rad. Transf., 113, 2334

Wang, L. 2005, ApJ, 635, L33

Wang, L., \& Wheeler, J. C. 2008, ARA\&A, 46, 433

Wang, X., Wang, L., Pain, R., Zhou, X., \& Li, Z. 2006, ApJ, 645, 488

Wang, X., Li, W., Filippenko, A. V., et al. 2008, ApJ, 677, 1060

Wegner, W. 2002, Baltic Astron., 11, 1

Weingartner, J. C., \& Draine, B. T. 2001, ApJ, 548, 296

Wesselius, P. R., van Duinen, R. J., de Jonge, A. R. W., et al. 1982, A\&AS, 49, 427

Whittet, D. C. B., \& van Breda I. G. 1978, A\&A, 66, 57

Whittet, D. C. B., Martin, P. G., Hough, J. H., et al. 1992, ApJ, 386, 562

Whittet, D. C. B., Gerakines, P. A., Carkner, A. L., et al. 1994, MNRAS, 268, 1

Whittet, D. C. B., Gerakines, P. A., Hough, J. H., \& Shenoy, S. S. 2001, ApJ, 547,872

Wilking, B. A., Lebofsky, M. J., Kemp, J. C., Martin, P. G., \& Rieke, G. H. 1980, ApJ, 235, 905

Wilking, B. A., Lebofsky, M. J., \& Rieke, G. H. 1982, AJ, 87, 695

Wolff, M. J., Nordsieck, K. H., \& Nook, M. A. 1996, AJ, 111, 856

Yang, Y., Wang, L., Baade, D., et al. 2017, ApJ, 834, 60

Zelaya, P., Clocchiatti, A., Baade, D., et al. 2017, ApJ, 836, 88 


\section{Appendix A: Standard stars}

\section{A.1. Standard stars with CAFOS}

Two unpolarized standard stars were observed with CAFOS: HD 144579 (2 epochs), and HD 90508 (one epoch), and two polarized standard stars, each at two epochs: HD 154445 and HD 43384.

We used the unpolarized standard stars to investigate possible instrumental effects. The observations were binned in $200 \AA$ bins, and the Stokes parameters, the polarization degree, and polarization angle were calculated as described in Sect. 3.3. Figure A.1 shows the derived Stokes parameters and polarization of the two unpolarized standard stars at three epochs compared to the instrumental polarization determined in Patat \& Taubenberger (2011). Our measurements show consistent values between the three epochs, with average $Q$ and $U$ vales of $0.07 \%$ and $0.004 \%$, respectively, in a wavelength range $3850-8650 \AA$, leading to an average polarization of $P \approx 0.10 \%$. The standard deviations are $\sigma_{Q} \approx 0.06 \%$, $\sigma_{U} \approx 0.11 \%$ and $\sigma_{P} \approx 0.07 \%$. The average uncertainty per $200 \AA$ bin is $\sim 0.03 \%$. Our values are more consistent with zero than the values determined in Patat \& Taubenberger (2011). They analyzed observations of the unpolarized star HD 14069 observed at 16 half-wave plate angles, and claimed average values of the instrumental Stokes parameters in a wavelength range above $4000 \AA$ of $\left\langle Q_{\text {ins }}\right\rangle=0.25 \pm 0.03 \%$ and $\left\langle U_{\text {ins }}\right\rangle=$ $-0.13 \pm 0.03 \%$, leading to an average polarization $P_{\text {ins }}=$ $0.28 \pm 0.03 \%$

Two polarized standard stars were observed with CAFOS, each at two epochs: HD 154445 and HD 43384. The calculated polarization spectra of HD 43384 are consistent with each other with an RMS of $\sim 0.04 \%$. Our Serkowski parameters (see Table C.2) are fully consistent with $p_{\max }=3.01 \pm 0.04 \%$ and $\lambda_{\max }=0.531 \pm 0.011 \mu \mathrm{m}$, determined in Hsu \& Breger (1982). The wavelength-dependent phase retardance variation of the half-wave plate deployed in CAFOS was quantified in Patat \& Taubenberger (2011). HD 43384 has a variable $\left(+0.6^{\circ} / 100 \mathrm{yr}\right)$, and slightly wavelength dependent $\left(+2.5 \pm 1.3^{\circ} / \mu \mathrm{m}\right)$ polarization position angle (Hsu \& Breger 1982). Therefore it is not the best standard star for HWP chromatism investigation. However, for comparison reasons, we used $\chi_{0}(V)=169.8 \pm 0.7$ degrees to compute the phase retardance variance and found that it is consistent with Patat \& Taubenberger (2011, see Fig. A.2). The average deviation of our phase retardance variation compared to Patat \& Taubenberger (2011) is $\sim+0.3$ degrees, which is within the errors of $\chi_{0}$.

Moreover, the two observed polarization spectra of HD 154445 are consistent with each other with an RMS of $0.07 \%$. The average $\lambda_{\max }=5579 \pm 11 \AA$ and $p_{\max }=3.64 \pm$ $0.01 \%$ matches the literature values $p_{\max }=3.66 \pm 0.01 \%$ and $\lambda_{\max }=5690 \pm 10 \AA$ (Wolff et al. 1996). Our average $\theta$ after the HWP chromatism correction is $89.2 \pm 0.4$ degree, which is similar to the literature values of $\theta_{V}=88.8 \pm 0.1$ (Schmidt et al. 1992), $\theta_{V}=90.1 \pm 0.1$ and $\theta_{\max }=88.3 \pm 0.1$ degrees (Hsu \& Breger 1982).

\section{A.2. Standard stars with AFOSC}

Because of lack of space in AFOSC, the Wollaston prism is inserted into the filter wheel in place of a filter for polarimetry purposes. During each observing run, it is therefore necessary to calibrate the instrument zero-point rotation angle using polarized standard stars.

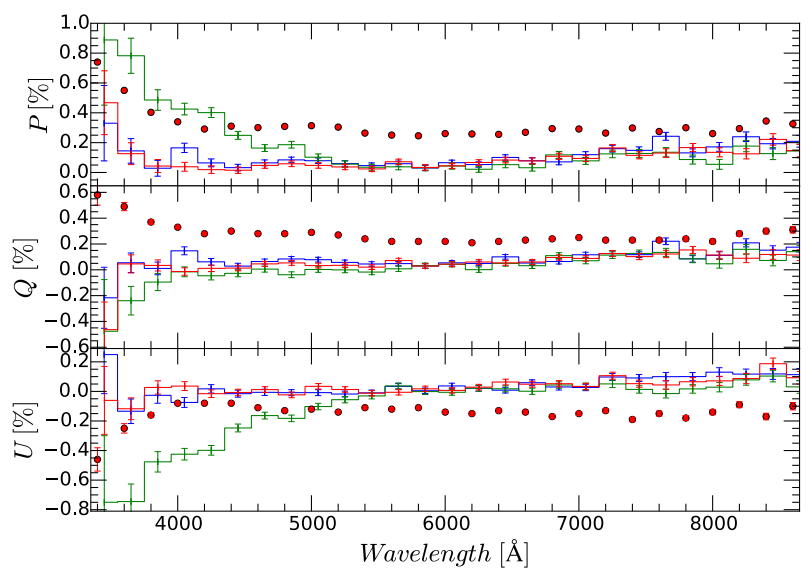

Fig. A.1. Unpolarized standard stars observed with CAFOS. HD 144579 was observed at two epochs, on 2015-04-30 at 00:51 UT (blue line) and 04:22 UT (green line). The red line indicates HD 90508 observed on 2015-04-29 at 21:28 UT. For comparison, the red dots indicate the instrumental polarization determined in Patat \& Taubenberger (2011).

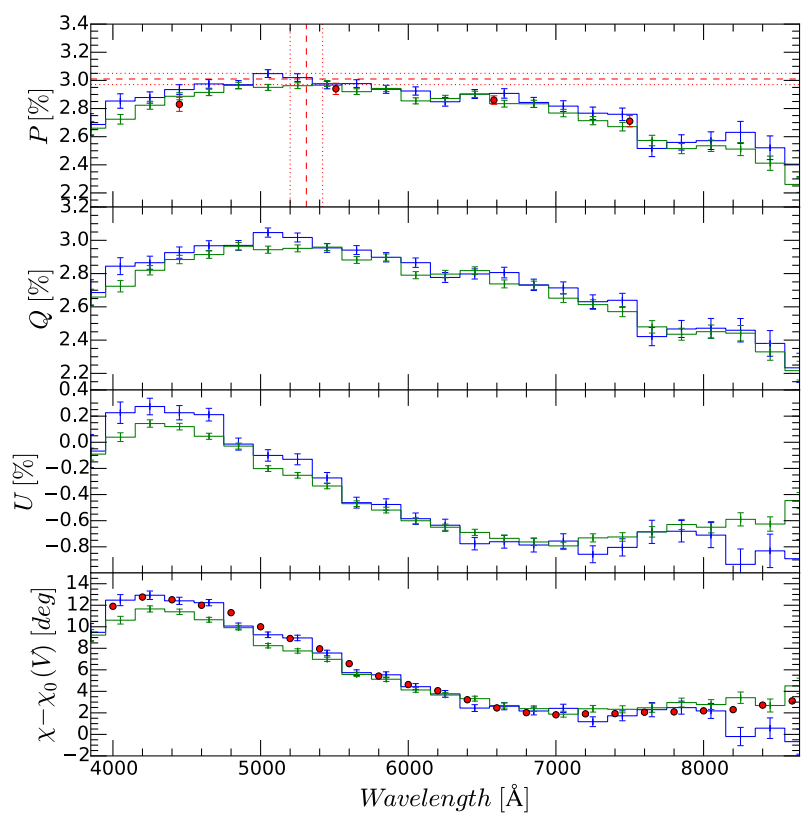

Fig. A.2. Polarized standard star HD 43384 observed with CAFOS at two epochs, on 2015-04-29 at 20:14 UT (green line) and 21:14 UT (blue line). The two epochs are consistent with each other with an RMS of $\sim 0.04 \%$. The red dashed lines in the polarization panel indicate $p_{\max }$ and $\lambda_{\max }$ and their errors (dotted line) determined by Hsu \& Breger (1982), and the red dots are their individual measurements. The red dots in the $\chi-\chi_{0}(V)$ panel indicate the phase retardance variance determined in Patat \& Taubenberger (2011).

We used observations of three unpolarized standard stars observed from 2015-02-09 to 2015-03-11 to investigate possible instrumental polarization of AFOSC: HD 90508 (two epochs), HD 39587 (two epochs), and HD 144579 (one epoch). They were all observed at four rotation angles of the adapter $(-45,0$, 45, and 90 degrees), except for one epoch of HD 90508, which was observed at only two rotation angles (0 and 90 degrees). Figure A. 3 shows the derived Stokes parameters $Q$ and $U$, and the polarization for all unpolarized standard stars. The black lines indicate the epochs observed at four rotation angles, and the blue line indicates HD 90508 observed at two rotation angles. The average stokes parameters at a wavelength range above 


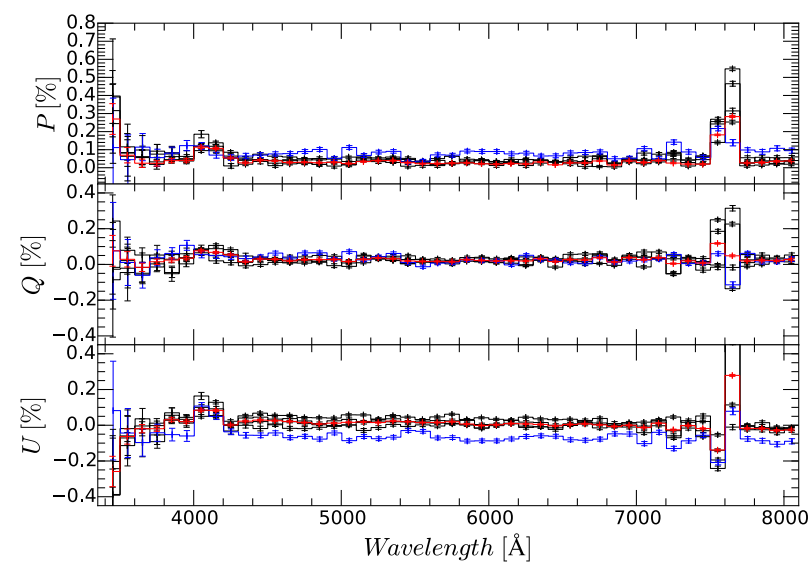

Fig. A.3. Unpolarized standard stars observed with AFOSC. The black lines indicate observations observed at four rotation angles, and the blue lines indicates HD 90508 observed at two rotation angles. The red line is the average of all measurements.

$3600 \AA$, excluding the range from $7500-7700 \AA$, which is contaminated by the strong telluric $7605.0 \AA \mathrm{O}_{2}$ line, are $0.03 \%$ and $-0.002 \%$ for $Q$ and $U$, respectively, leading to a polarization of $0.05 \%$, with a standard deviation of $0.05 \%$.

When the observations performed with the adapter rotation angles of -45 and 45 degrees are ignored and the polarization is calculated using only the adapter rotation angles of 0 and 90 degrees for all five epochs of the three standard stars, the average stokes parameters are $0.05 \%$ and $-0.04 \%$ for $Q$ and $U$, respectively, leading to a polarization of $0.08 \%$, with a standard deviation of $0.10 \%$.

HD 185395, observed on 2016-08-02, is also consistent with zero, with an average polarization above $3600 \AA$, excluding the range from $7500-7700 \AA$, of $0.08 \%$ and an RMS of $0.06 \%$.

Three polarized standard stars were observed with AFOSC: HD 43384 (three epochs at two different runs), HD 21291 (one epoch), and HD 198478 (one epoch), all at four rotation angles of the adapter. However, because most of the science data were taken only with two rotation angles, we used only two rotation angles for consistency.

Figure A.4 shows HD 43384 at three different epochs. Although the shapes of the polarization spectra are similar, that is, $\lambda_{\max }$ and $K$ of the Serkowski fit are similar to each other, the peak polarization values, $p_{\max }$, are not consistent and range from $\sim 2.92 \%$ to $\sim 3.19 \%$ (see Table C.3), while the literature value is $p_{\max }=3.01 \pm 0.04 \%$ (Hsu \& Breger 1982).

For HD 21291, our determined peak polarization value is lower than the literature value. By fitting the Serkowski curve, we find $\lambda_{\max }=5166 \pm 27, p_{\max }=2.95 \pm 0.01 \%$, while the literature values are $p_{\max } \sim 3.53 \pm 0.02 \%$ and $\lambda_{\max }=5210 \pm 30 \AA$ (Hsu \& Breger 1982).

HD 198478 was observed on 2016-08-02. Our determined peak polarization value is almost consistent with the literature value. By fitting the Serkowski curve, we find $\lambda_{\max }=5132 \pm 42$, $p_{\max }=2.76 \pm 0.01 \%$, while the literature values are $p_{\max } \sim 2.72 \pm$ $0.02 \%$ and $\lambda_{\max }=5220 \pm 80 \AA$ (Hsu \& Breger 1982).

We used polarized standard stars to determine the correction of the instrument rotation angle zero-point for each of the three runs separately.

During the first run (2015-02-09), two stars were observed: HD 43384 (two epochs) and HD 21291 (one epoch). Using the

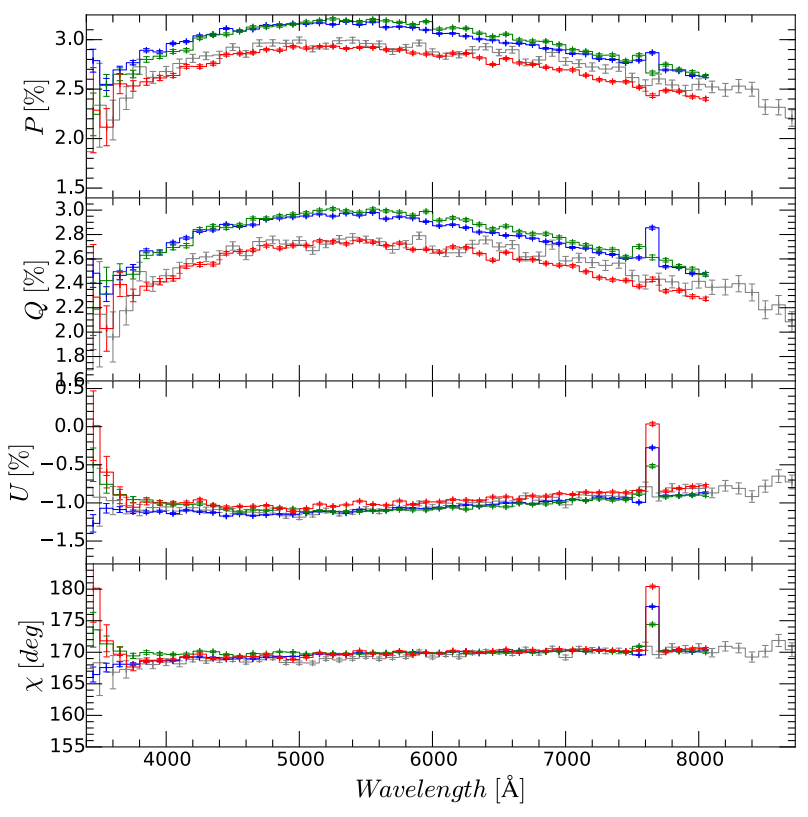

Fig. A.4. Polarized standard star HD 43384 observed in three different nights with AFOSC. The peak polarization values, $p_{\max }$, are not fully consistent and range from $\sim 2.92 \%$ to $\sim 3.19 \%$. For reference, the gray line indicates HD 43384 observed with CAFOS.

literature values of $\chi_{V}=169.8 \pm 0.7$ degrees for HD 43384, and $\chi_{V}=116.6 \pm 0.2$ degrees for HD 21291, we calculated a weighted average of the offset $\Delta \theta_{V}=136.4 \pm 0.3$ degrees.

During the second run (2015-03-09), HD 43384 was observed at one epoch, from which we calculated the instrument rotation angle zero-point offset $\Delta \theta_{V}=134.0 \pm 0.8$ degrees.

Finally, from HD 198478 observed during the third run (2016-08-02), we calculated $\Delta \theta_{V}=138.3 \pm 0.4$ degrees.

\section{Appendix B: Serkowski fit to SNe la}

We determined the Serkowski parameters of the sodium sample in Zelaya et al. (2017) by fitting Eq. (1) to the data shown in their Fig. 1. The polarized lines were excluded from the wavelength range. The results are given in Table B.1.

Table B.1. Serkowski paramaters of the SNe Ia sodium-sample (Zelaya et al. 2017).

\begin{tabular}{llll}
\hline \hline SN Name & $\lambda_{\max }(\AA)$ & $p_{\max }(\%)$ & $K$ \\
\hline SN 2007le & $3967 \pm 494$ & $1.93 \pm 0.09$ & $1.73 \pm 0.87$ \\
SN 2010ev & $4408 \pm 114$ & $1.88 \pm 0.02$ & $1.89 \pm 0.23$ \\
SN 2007fb & $3821 \pm 447$ & $0.76 \pm 0.03$ & $1.13 \pm 0.46$ \\
SN 2003W & $3996 \pm 371$ & $0.78 \pm 0.04$ & $2.56 \pm 1.03$ \\
SN 2007af & $7409 \pm 537$ & $0.64 \pm 0.01$ & $1.24 \pm 0.35$ \\
SN 2002fk & $4403 \pm 460$ & $0.40 \pm 0.01$ & $0.57 \pm 0.35$ \\
SN 2002bo & $3525 \pm 137$ & $1.11 \pm 0.05$ & $3.95 \pm 0.49$ \\
SN 2011ae & $4256 \pm 295$ & $0.33 \pm 0.01$ & $3.56 \pm 1.87$ \\
SN 2005hk & $6731 \pm 2116$ & $0.17 \pm 0.02$ & $-1.36 \pm 1.51$
\end{tabular}

Notes. ${ }^{(a)}$ Because of a low polarization degree and low S/N, a good fit with the Serkowski curve was not possible. 
Appendix C: Individual observations

Table C.1. Individual epochs with FORS2.

\begin{tabular}{|c|c|c|c|c|c|c|c|c|c|c|}
\hline \multirow[b]{2}{*}{ Name } & \multirow[b]{2}{*}{ Filter } & \multirow[b]{2}{*}{ Epoch } & \multirow[b]{2}{*}{ Passband } & \multirow[b]{2}{*}{$P(\%)$} & \multirow[b]{2}{*}{$P_{Q}(\%)$} & \multirow[b]{2}{*}{$P_{U}(\%)$} & \multirow[b]{2}{*}{$\theta\left(^{\circ}\right)$} & & erkowski cur & \\
\hline & & & & & & & & $\lambda_{\max }(\AA)$ & $P_{\max }(\%)$ & $K$ \\
\hline HD 141318 & free & 2014-10-10 00:23:57 & & & & & & $5827 \pm 40$ & $2.43 \pm 0.01$ & $1.11 \pm 0.06$ \\
\hline & & & $\mathrm{B}$ & $2.28 \pm 0.02$ & $-0.41 \pm 0.01$ & $2.24 \pm 0.02$ & $50.2 \pm 0.2$ & & & \\
\hline & & & V & $2.43 \pm 0.01$ & $-0.52 \pm 0.01$ & $2.38 \pm 0.01$ & $51.2 \pm 0.2$ & & & \\
\hline & & & $\mathrm{R}$ & $2.40 \pm 0.01$ & $-0.55 \pm 0.01$ & $2.34 \pm 0.01$ & $51.7 \pm 0.1$ & & & \\
\hline & & & $\mathrm{I}$ & $2.18 \pm 0.01$ & $-0.50 \pm 0.01$ & $2.12 \pm 0.01$ & $51.7 \pm 0.1$ & & & \\
\hline HD 141318 & free & 2014-10-10 00:27:27 & & & & & & $5770 \pm 23$ & $2.46 \pm 0.01$ & $1.29 \pm 0.04$ \\
\hline & & & $\mathrm{B}$ & $2.30 \pm 0.01$ & $-0.40 \pm 0.01$ & $2.26 \pm 0.01$ & $50.0 \pm 0.1$ & & & \\
\hline & & & V & $2.46 \pm 0.01$ & $-0.52 \pm 0.01$ & $2.40 \pm 0.01$ & $51.1 \pm 0.1$ & & & \\
\hline & & & $\mathrm{R}$ & $2.41 \pm 0.01$ & $-0.55 \pm 0.01$ & $2.35 \pm 0.01$ & $51.6 \pm 0.1$ & & & \\
\hline & & & I & $2.15 \pm 0.01$ & $-0.48 \pm 0.01$ & $2.10 \pm 0.01$ & $51.4 \pm 0.1$ & & & \\
\hline HD 141318 & GG435 & 2014-10-10 00:37:56 & & & & & & $5544 \pm 57$ & $2.43 \pm 0.01$ & $1.24 \pm 0.08$ \\
\hline & & & $\mathrm{B}$ & $2.35 \pm 0.02$ & $-0.49 \pm 0.02$ & $2.30 \pm 0.02$ & $51.0 \pm 0.2$ & & & \\
\hline & & & V & $2.44 \pm 0.01$ & $-0.50 \pm 0.01$ & $2.39 \pm 0.01$ & $50.9 \pm 0.1$ & & & \\
\hline & & & $\mathrm{R}$ & $2.36 \pm 0.01$ & $-0.50 \pm 0.01$ & $2.31 \pm 0.01$ & $51.1 \pm 0.1$ & & & \\
\hline & & & $\mathrm{I}$ & $2.06 \pm 0.01$ & $-0.39 \pm 0.01$ & $2.02 \pm 0.01$ & $50.6 \pm 0.1$ & & & \\
\hline HD 141318 & GG435 & 2014-10-10 00:46:02 & & & & & & $5851 \pm 79$ & $2.38 \pm 0.01$ & $0.81 \pm 0.08$ \\
\hline & & & $\mathrm{B}$ & $2.33 \pm 0.02$ & $-0.46 \pm 0.02$ & $2.28 \pm 0.02$ & $50.7 \pm 0.2$ & & & \\
\hline & & & V & $2.40 \pm 0.01$ & $-0.44 \pm 0.01$ & $2.36 \pm 0.01$ & $50.3 \pm 0.1$ & & & \\
\hline & & & $\mathrm{R}$ & $2.37 \pm 0.01$ & $-0.46 \pm 0.01$ & $2.32 \pm 0.01$ & $50.6 \pm 0.1$ & & & \\
\hline & & & $\mathrm{I}$ & $2.20 \pm 0.01$ & $-0.46 \pm 0.01$ & $2.15 \pm 0.01$ & $51.0 \pm 0.1$ & & & \\
\hline HD 152245 & free & 2014-10-13 00:22:31 & & & & & & $6360 \pm 63$ & $0.93 \pm 0.01$ & $1.32 \pm 0.12$ \\
\hline & & & $\mathrm{B}$ & $0.80 \pm 0.01$ & $-0.10 \pm 0.01$ & $0.79 \pm 0.01$ & $48.4 \pm 0.4$ & & & \\
\hline & & & V & $0.91 \pm 0.01$ & $-0.10 \pm 0.01$ & $0.90 \pm 0.01$ & $48.3 \pm 0.3$ & & & \\
\hline & & & $\mathrm{R}$ & $0.92 \pm 0.01$ & $-0.10 \pm 0.01$ & $0.92 \pm 0.01$ & $48.2 \pm 0.2$ & & & \\
\hline & & & I & $0.84 \pm 0.01$ & $-0.06 \pm 0.01$ & $0.84 \pm 0.01$ & $47.2 \pm 0.2$ & & & \\
\hline HD 152245 & GG435 & 2015-02-06 08:26:24 & & & & & & $6174 \pm 64$ & $1.01 \pm 0.01$ & $1.25 \pm 0.12$ \\
\hline & & & $\mathrm{B}$ & $0.92 \pm 0.01$ & $0.18 \pm 0.01$ & $0.90 \pm 0.01$ & $39.3 \pm 0.4$ & & & \\
\hline & & & V & $0.98 \pm 0.01$ & $0.24 \pm 0.01$ & $0.95 \pm 0.01$ & $38.0 \pm 0.2$ & & & \\
\hline & & & $\mathrm{R}$ & $0.98 \pm 0.01$ & $0.25 \pm 0.01$ & $0.94 \pm 0.01$ & $37.6 \pm 0.1$ & & & \\
\hline & & & I & $0.88 \pm 0.01$ & $0.26 \pm 0.01$ & $0.84 \pm 0.01$ & $36.4 \pm 0.2$ & & & \\
\hline HD 152245 & GG435 & 2015-02-06 08:31:36.880 & & & & & & $6465 \pm 66$ & $1.0 \pm 0.01$ & $1.13 \pm 0.11$ \\
\hline & & & $\mathrm{B}$ & $0.88 \pm 0.01$ & $0.22 \pm 0.01$ & $0.85 \pm 0.01$ & $37.9 \pm 0.5$ & & & \\
\hline & & & V & $0.95 \pm 0.01$ & $0.28 \pm 0.01$ & $0.91 \pm 0.01$ & $36.4 \pm 0.2$ & & & \\
\hline & & & $\mathrm{R}$ & $0.97 \pm 0.01$ & $0.30 \pm 0.01$ & $0.92 \pm 0.01$ & $36.1 \pm 0.1$ & & & \\
\hline & & & $\mathrm{I}$ & $0.91 \pm 0.01$ & $0.30 \pm 0.01$ & $0.85 \pm 0.01$ & $35.3 \pm 0.2$ & & & \\
\hline HD 54439 & free & 2014-10-27Т05:39:01 & & & & & & $4802 \pm 196$ & $0.8 \pm 0.01$ & $0.82 \pm 0.13$ \\
\hline & & & $\mathrm{B}$ & $0.76 \pm 0.01$ & $0.04 \pm 0.01$ & $-0.76 \pm 0.01$ & $136.6 \pm 0.5$ & & & \\
\hline & & & V & $0.76 \pm 0.01$ & $0.09 \pm 0.01$ & $-0.76 \pm 0.01$ & $138.2 \pm 0.4$ & & & \\
\hline & & & $\mathrm{R}$ & $0.72 \pm 0.01$ & $0.11 \pm 0.01$ & $-0.71 \pm 0.01$ & $139.3 \pm 0.2$ & & & \\
\hline & & & $\mathrm{I}$ & $0.64 \pm 0.01$ & $0.07 \pm 0.01$ & $-0.64 \pm 0.01$ & $138.2 \pm 0.4$ & & & \\
\hline HD 54439 & GG435 & 2014-10-27 05:50:15 & & & & & & $4756 \pm 268$ & $0.79 \pm 0.01$ & $1.11 \pm 0.22$ \\
\hline & & & $\mathrm{B}$ & $0.74 \pm 0.02$ & $0.10 \pm 0.02$ & $-0.73 \pm 0.02$ & $139.0 \pm 0.7$ & & & \\
\hline & & & $\mathrm{V}$ & $0.75 \pm 0.01$ & $0.11 \pm 0.01$ & $-0.74 \pm 0.01$ & $139.3 \pm 0.4$ & & & \\
\hline & & & $\mathrm{R}$ & $0.69 \pm 0.01$ & $0.15 \pm 0.01$ & $-0.68 \pm 0.01$ & $141.2 \pm 0.3$ & & & \\
\hline & & & $\mathrm{I}$ & $0.58 \pm 0.01$ & $0.15 \pm 0.01$ & $-0.56 \pm 0.01$ & $142.4 \pm 0.4$ & & & \\
\hline HD 73420 & free & 2014-10-27 06:04:31 & & & & & & $6756 \pm 323$ & $0.55 \pm 0.01$ & $0.65 \pm 0.23$ \\
\hline & & & $\mathrm{B}$ & $0.35 \pm 0.01$ & $0.35 \pm 0.01$ & $-0.04 \pm 0.02$ & $176.4 \pm 1.0$ & & & \\
\hline & & & V & $0.37 \pm 0.01$ & $0.36 \pm 0.01$ & $-0.10 \pm 0.01$ & $172.5 \pm 0.7$ & & & \\
\hline & & & $\mathrm{R}$ & $0.36 \pm 0.01$ & $0.35 \pm 0.01$ & $-0.08 \pm 0.01$ & $173.4 \pm 0.4$ & & & \\
\hline & & & $\mathrm{I}$ & $0.33 \pm 0.01$ & $0.32 \pm 0.01$ & $-0.08 \pm 0.01$ & $172.5 \pm 0.6$ & & & \\
\hline HD 73420 & GG435 & 2014-10-27 06:41:43 & & & & & & $6482 \pm 150$ & $0.42 \pm 0.01$ & $1.22 \pm 0.28$ \\
\hline & & & B & $0.35 \pm 0.02$ & $0.34 \pm 0.02$ & $-0.08 \pm 0.02$ & $173.3 \pm 1.3$ & & & \\
\hline & & & V & $0.38 \pm 0.01$ & $0.35 \pm 0.01$ & $-0.15 \pm 0.01$ & $168.4 \pm 0.6$ & & & \\
\hline & & & $\mathrm{R}$ & $0.38 \pm 0.01$ & $0.36 \pm 0.01$ & $-0.14 \pm 0.01$ & $169.2 \pm 0.4$ & & & \\
\hline & & & $\mathrm{I}$ & $0.30 \pm 0.01$ & $0.28 \pm 0.01$ & $-0.11 \pm 0.01$ & $168.8 \pm 0.6$ & & & \\
\hline HD 78785 & free & 2014-11-14 07:04:31 & & & & & & $5771 \pm 10$ & $3.96 \pm 0.01$ & $1.22 \pm 0.02$ \\
\hline & & & $\mathrm{B}$ & $3.64 \pm 0.01$ & $3.39 \pm 0.01$ & $1.30 \pm 0.01$ & $10.5 \pm 0.1$ & & & \\
\hline & & & V & $3.88 \pm 0.01$ & $3.63 \pm 0.01$ & $1.37 \pm 0.01$ & $10.3 \pm 0.1$ & & & \\
\hline & & & $\mathrm{R}$ & $3.80 \pm 0.01$ & $3.53 \pm 0.01$ & $1.39 \pm 0.01$ & $10.8 \pm 0.1$ & & & \\
\hline & & & $\mathrm{I}$ & $3.40 \pm 0.01$ & $3.16 \pm 0.01$ & $1.26 \pm 0.01$ & $10.9 \pm 0.1$ & & & \\
\hline HD 78785 & GG435 & 2014-11-14 06:54:19 & & & & & & $5766 \pm 16$ & $3.97 \pm 0.01$ & $1.18 \pm 0.02$ \\
\hline & & & $\mathrm{B}$ & $3.73 \pm 0.02$ & $3.48 \pm 0.02$ & $1.35 \pm 0.02$ & $10.6 \pm 0.1$ & & & \\
\hline & & & $\mathrm{V}$ & $3.89 \pm 0.01$ & $3.65 \pm 0.01$ & $1.34 \pm 0.01$ & $10.0 \pm 0.1$ & & & \\
\hline & & & $\mathrm{R}$ & $3.81 \pm 0.01$ & $3.55 \pm 0.01$ & $1.38 \pm 0.01$ & $10.6 \pm 0.1$ & & & \\
\hline & & & $\mathrm{I}$ & $3.41 \pm 0.01$ & $3.18 \pm 0.01$ & $1.24 \pm 0.01$ & $10.6 \pm 0.1$ & & & \\
\hline HD 78785 & GG435 & 2014-11-14 07:13:33 & & & & & & $5754 \pm 17$ & $3.94 \pm 0.01$ & $1.18 \pm 0.02$ \\
\hline & & & B & $3.71 \pm 0.02$ & $3.45 \pm 0.02$ & $1.37 \pm 0.02$ & $10.8 \pm 0.1$ & & & \\
\hline & & & V & $3.86 \pm 0.01$ & $3.62 \pm 0.01$ & $1.34 \pm 0.01$ & $10.2 \pm 0.1$ & & & \\
\hline & & & $\mathrm{R}$ & $3.78 \pm 0.01$ & $3.52 \pm 0.01$ & $1.38 \pm 0.01$ & $10.7 \pm 0.1$ & & & \\
\hline & & & $\mathrm{I}$ & $3.38 \pm 0.01$ & $3.15 \pm 0.01$ & $1.24 \pm 0.01$ & $10.8 \pm 0.1$ & & & \\
\hline
\end{tabular}

Notes. The errors in this table are statistical only, while the root-mean-square of the Stokes $Q$ and $U$ is $\sim 0.05 \%$. ${ }^{(a)}$ The constant polarization curve of this star could not be fitted well with a Serkowski curve. ${ }^{(b)}$ The polarization angle could not be determined due to low polarization degree. 
A. Cikota et al.: Spectropolarimetry of Galactic stars with anomalous extinction sightlines

Table C.1. continued.

\begin{tabular}{|c|c|c|c|c|c|c|c|c|c|c|}
\hline \multirow[b]{2}{*}{ Name } & \multirow[b]{2}{*}{ Filter } & \multirow[b]{2}{*}{ Epoch } & \multirow[b]{2}{*}{ Passband } & \multirow[b]{2}{*}{$P(\%)$} & \multirow[b]{2}{*}{$P_{Q}(\%)$} & \multirow[b]{2}{*}{$P_{U}(\%)$} & \multirow[b]{2}{*}{$\theta\left(^{\circ}\right)$} & \multicolumn{3}{|c|}{ Serkowski curve } \\
\hline & & & & & & & & $\lambda_{\max }(\AA)$ & $P_{\max }(\%)$ & $K$ \\
\hline \multirow[t]{5}{*}{ HD 96042} & free & 2014-12-21 08:14:55 & & & & & & $4816 \pm 255$ & $0.58 \pm 0.01$ & $0.93 \pm 0.19$ \\
\hline & & & B & $0.59 \pm 0.01$ & $-0.38 \pm 0.01$ & $-0.45 \pm 0.01$ & $115.1 \pm 0.5$ & & & \\
\hline & & & V & $0.60 \pm 0.01$ & $-0.40 \pm 0.01$ & $-0.45 \pm 0.01$ & $114.0 \pm 0.4$ & & & \\
\hline & & & $\mathrm{R}$ & $0.57 \pm 0.01$ & $-0.38 \pm 0.01$ & $-0.42 \pm 0.01$ & $114.0 \pm 0.3$ & & & \\
\hline & & & $\mathrm{I}$ & $0.51 \pm 0.01$ & $-0.34 \pm 0.01$ & $-0.38 \pm 0.01$ & $114.2 \pm 0.4$ & & & \\
\hline \multirow[t]{5}{*}{ HD 96042} & free & 2014-12-21 08:22:47 & & & & & & $4850 \pm 245$ & $0.60 \pm 0.01$ & $0.90 \pm 0.18$ \\
\hline & & & $\mathrm{B}$ & $0.60 \pm 0.01$ & $-0.39 \pm 0.01$ & $-0.46 \pm 0.01$ & $114.7 \pm 0.5$ & & & \\
\hline & & & V & $0.62 \pm 0.01$ & $-0.41 \pm 0.01$ & $-0.46 \pm 0.01$ & $114.1 \pm 0.4$ & & & \\
\hline & & & $\mathrm{R}$ & $0.59 \pm 0.01$ & $-0.39 \pm 0.01$ & $-0.44 \pm 0.01$ & $114.2 \pm 0.3$ & & & \\
\hline & & & I & $0.54 \pm 0.01$ & $-0.36 \pm 0.01$ & $-0.40 \pm 0.01$ & $114.1 \pm 0.4$ & & & \\
\hline \multirow[t]{5}{*}{ HD 96042} & GG435 & 2015-01-02 07:25:12 & & & & & & $3494 \pm 955$ & $0.64 \pm 0.05$ & $0.51 \pm 0.24$ \\
\hline & & & B & $0.62 \pm 0.02$ & $-0.34 \pm 0.02$ & $-0.52 \pm 0.02$ & $118.5 \pm 0.8$ & & & \\
\hline & & & V & $0.60 \pm 0.01$ & $-0.36 \pm 0.01$ & $-0.49 \pm 0.01$ & $116.9 \pm 0.5$ & & & \\
\hline & & & $\mathrm{R}$ & $0.56 \pm 0.01$ & $-0.33 \pm 0.01$ & $-0.46 \pm 0.01$ & $117.2 \pm 0.3$ & & & \\
\hline & & & $\mathrm{I}$ & $0.51 \pm 0.01$ & $-0.32 \pm 0.01$ & $-0.40 \pm 0.01$ & $115.8 \pm 0.5$ & & & \\
\hline \multirow[t]{5}{*}{ HD 96042} & GG435 & 2015-01-02 07:29:02 & & & & & & $5116 \pm 242$ & $0.59 \pm 0.01$ & $1.43 \pm 0.30$ \\
\hline & & & $\mathrm{B}$ & $0.59 \pm 0.02$ & $-0.35 \pm 0.02$ & $-0.48 \pm 0.02$ & $117.0 \pm 1.0$ & & & \\
\hline & & & $\mathrm{V}$ & $0.62 \pm 0.01$ & $-0.39 \pm 0.01$ & $-0.47 \pm 0.01$ & $115.1 \pm 0.5$ & & & \\
\hline & & & $\mathrm{R}$ & $0.58 \pm 0.01$ & $-0.36 \pm 0.01$ & $-0.45 \pm 0.01$ & $115.4 \pm 0.3$ & & & \\
\hline & & & I & $0.49 \pm 0.01$ & $-0.31 \pm 0.01$ & $-0.37 \pm 0.01$ & $115.0 \pm 0.5$ & & & \\
\hline HD 152853 & free & $2015-02-03$ 07:45:45 & & & & & & $5804 \pm 75$ & $1.78 \pm 0.02$ & $1.35 \pm 0.14$ \\
\hline & & & B & $1.57 \pm 0.01$ & $1.19 \pm 0.01$ & $1.02 \pm 0.01$ & $20.3 \pm 0.2$ & & & \\
\hline & & & $\mathrm{V}$ & $1.71 \pm 0.01$ & $1.29 \pm 0.01$ & $1.13 \pm 0.01$ & $20.6 \pm 0.2$ & & & \\
\hline & & & $\mathrm{R}$ & $1.71 \pm 0.01$ & $1.26 \pm 0.01$ & $1.15 \pm 0.01$ & $21.1 \pm 0.1$ & & & \\
\hline & & & I & $1.42 \pm 0.01$ & $1.04 \pm 0.01$ & $0.97 \pm 0.01$ & $21.5 \pm 0.2$ & & & \\
\hline HD 152853 & GG435 & 2015-02-03 08:03:39 & & & & & & $5619 \pm 67$ & $1.69 \pm 0.01$ & $1.11 \pm 0.08$ \\
\hline & & & $\mathrm{B}$ & $1.59 \pm 0.02$ & $1.18 \pm 0.02$ & $1.06 \pm 0.02$ & $21.0 \pm 0.3$ & & & \\
\hline & & & $\mathrm{V}$ & $1.64 \pm 0.01$ & $1.21 \pm 0.01$ & $1.10 \pm 0.01$ & $21.2 \pm 0.2$ & & & \\
\hline & & & $\mathrm{R}$ & $1.60 \pm 0.01$ & $1.14 \pm 0.01$ & $1.12 \pm 0.01$ & $22.3 \pm 0.1$ & & & \\
\hline & & & $\mathrm{I}$ & $1.41 \pm 0.01$ & $0.98 \pm 0.01$ & $1.00 \pm 0.01$ & $22.8 \pm 0.1$ & & & \\
\hline HD $137569^{a, b}$ & free & 2015-02-03 08:31:58 & & & & & & $\ldots$ & $\ldots$ & $\ldots$ \\
\hline & & & $\mathrm{B}$ & $0.11 \pm 0.01$ & $-0.09 \pm 0.01$ & $0.05 \pm 0.01$ & $\ldots$ & & & \\
\hline & & & V & $0.13 \pm 0.01$ & $-0.11 \pm 0.01$ & $0.07 \pm 0.01$ & $\ldots$ & & & \\
\hline & & & $\mathrm{R}$ & $0.11 \pm 0.01$ & $-0.10 \pm 0.01$ & $0.06 \pm 0.01$ & $\ldots$ & & & \\
\hline & & & $\mathrm{I}$ & $0.09 \pm 0.01$ & $-0.08 \pm 0.01$ & $0.05 \pm 0.01$ & $\ldots$ & & & \\
\hline HD $137569^{a, b}$ & free & 2015-02-03Т08:43:51 & & & & & & $\ldots$ & $\ldots$ & $\ldots$ \\
\hline & & & B & $0.11 \pm 0.01$ & $-0.11 \pm 0.01$ & $0.04 \pm 0.01$ & $\ldots$ & & & \\
\hline & & & $\mathrm{V}$ & $0.12 \pm 0.01$ & $-0.12 \pm 0.01$ & $0.04 \pm 0.01$ & $\ldots$ & & & \\
\hline & & & $\mathrm{R}$ & $0.11 \pm 0.01$ & $-0.10 \pm 0.01$ & $0.05 \pm 0.01$ & $\ldots$ & & & \\
\hline & & & $\mathrm{I}$ & $0.09 \pm 0.01$ & $-0.06 \pm 0.01$ & $0.06 \pm 0.01$ & $\ldots$ & & & \\
\hline HD $137569^{a, b}$ & GG435 & 2015-02-06 07:58:28 & & & & & & $\ldots$ & $\ldots$ & $\ldots$ \\
\hline & & & $\mathrm{B}$ & $0.10 \pm 0.03$ & $0.08 \pm 0.03$ & $0.06 \pm 0.03$ & $\ldots$ & & & \\
\hline & & & V & $0.07 \pm 0.02$ & $0.07 \pm 0.02$ & $0.02 \pm 0.02$ & $\ldots$ & & & \\
\hline & & & $\mathrm{R}$ & $0.11 \pm 0.01$ & $0.10 \pm 0.01$ & $0.03 \pm 0.01$ & $\ldots$ & & & \\
\hline & & & $\mathrm{I}$ & $0.13 \pm 0.01$ & $0.11 \pm 0.01$ & $0.06 \pm 0.01$ & $\ldots$ & & & \\
\hline HD $137569^{a, b}$ & GG435 & 2015-02-06 08:45:46 & & & & & & $\ldots$ & $\ldots$ & $\ldots$ \\
\hline & & & B & $0.47 \pm 0.02$ & $-0.21 \pm 0.02$ & $0.42 \pm 0.02$ & $\ldots$ & & & \\
\hline & & & $\mathrm{V}$ & $0.39 \pm 0.01$ & $-0.15 \pm 0.01$ & $0.36 \pm 0.01$ & $\ldots$ & & & \\
\hline & & & $\mathrm{R}$ & $0.31 \pm 0.01$ & $-0.14 \pm 0.01$ & $0.27 \pm 0.01$ & $\ldots$ & & & \\
\hline & & & $\mathrm{I}$ & $0.19 \pm 0.01$ & $-0.12 \pm 0.01$ & $0.15 \pm 0.01$ & $\ldots$ & & & \\
\hline HD $137569^{a, b}$ & GG435 & 2015-02-06 08:49:35 & & & & & & $\ldots$ & $\ldots$ & $\ldots$ \\
\hline & & & B & $0.09 \pm 0.02$ & $-0.09 \pm 0.02$ & $0.02 \pm 0.02$ & $\ldots$ & & & \\
\hline & & & $\mathrm{V}$ & $0.06 \pm 0.01$ & $-0.04 \pm 0.01$ & $0.04 \pm 0.01$ & $\ldots$ & & & \\
\hline & & & $\mathrm{R}$ & $0.04 \pm 0.01$ & $-0.02 \pm 0.01$ & $0.03 \pm 0.01$ & $\ldots$ & & & \\
\hline & & & $\mathrm{I}$ & $0.03 \pm 0.01$ & $0.02 \pm 0.01$ & $0.03 \pm 0.01$ & $\ldots$ & & & \\
\hline HD $137569^{a, b}$ & GG435 & 2015-02-06 08:58:20 & & & & & & $\ldots$ & . & $\ldots$ \\
\hline & & & $\mathrm{B}$ & $0.47 \pm 0.02$ & $-0.21 \pm 0.02$ & $-0.42 \pm 0.02$ & $\ldots$ & & & \\
\hline & & & $\mathrm{V}$ & $0.40 \pm 0.01$ & $-0.18 \pm 0.01$ & $-0.36 \pm 0.01$ & $\ldots$ & & & \\
\hline & & & $\mathrm{R}$ & $0.34 \pm 0.01$ & $-0.11 \pm 0.01$ & $-0.32 \pm 0.01$ & $\ldots$ & & & \\
\hline & & & $\mathrm{I}$ & $0.30 \pm 0.01$ & $-0.05 \pm 0.01$ & $-0.29 \pm 0.01$ & $\ldots$ & & & \\
\hline
\end{tabular}


Table C.2. Individual epochs with CAFOS

\begin{tabular}{|c|c|c|c|c|c|c|c|c|c|c|}
\hline \multirow[b]{2}{*}{ Name } & \multirow[b]{2}{*}{ Filter } & \multirow[b]{2}{*}{ Epoch } & \multirow[b]{2}{*}{ Passband } & \multirow[b]{2}{*}{$P(\%)$} & \multirow[b]{2}{*}{$P_{Q}(\%)$} & \multirow[b]{2}{*}{$P_{U}(\%)$} & \multirow[b]{2}{*}{$\theta\left(^{\circ}\right)$} & \multicolumn{3}{|c|}{ Serkowski curve } \\
\hline & & & & & & & & $\lambda_{\max }(\AA)$ & $P_{\max }(\%)$ & $K$ \\
\hline \multirow[t]{4}{*}{$B D+23 d 3762$} & free & 2015-04-30 03:09:40 & & & & & & $4964.8 \pm 60.9$ & $2.23 \pm 0.01$ & $0.92 \pm 0.06$ \\
\hline & & & $\mathrm{B}$ & $2.20 \pm 0.01$ & $1.83 \pm 0.01$ & $1.21 \pm 0.02$ & $16.7 \pm 0.2$ & & & \\
\hline & & & $\mathrm{V}$ & $2.19 \pm 0.01$ & $1.76 \pm 0.01$ & $1.31 \pm 0.02$ & $18.3 \pm 0.2$ & & & \\
\hline & & & $\mathrm{R}$ & $2.07 \pm 0.01$ & $1.62 \pm 0.01$ & $1.28 \pm 0.01$ & $19.2 \pm 0.1$ & & & \\
\hline \multirow[t]{4}{*}{$\mathrm{BD}+45 \mathrm{~d} 3341$} & free & 2015-04-30 02:06:40 & & & & & & $5046.5 \pm 72.6$ & $3.09 \pm 0.01$ & $0.83 \pm 0.08$ \\
\hline & & & B & $3.02 \pm 0.02$ & $-0.19 \pm 0.01$ & $3.02 \pm 0.02$ & $46.8 \pm 0.2$ & & & \\
\hline & & & V & $3.06 \pm 0.02$ & $-0.31 \pm 0.01$ & $3.04 \pm 0.02$ & $47.9 \pm 0.1$ & & & \\
\hline & & & $\mathrm{R}$ & $2.90 \pm 0.01$ & $-0.31 \pm 0.01$ & $2.88 \pm 0.01$ & $48.0 \pm 0.1$ & & & \\
\hline \multirow[t]{4}{*}{$\mathrm{BD}+45 \mathrm{~d} 3341$} & free & 2015-04-30 02:34:01 & & & & & & $5192.5 \pm 34.0$ & $3.01 \pm 0.01$ & $1.09 \pm 0.06$ \\
\hline & & & B & $2.93 \pm 0.01$ & $-0.20 \pm 0.01$ & $2.92 \pm 0.01$ & $47.0 \pm 0.1$ & & & \\
\hline & & & $\mathrm{V}$ & $2.97 \pm 0.01$ & $-0.30 \pm 0.01$ & $2.96 \pm 0.01$ & $47.9 \pm 0.1$ & & & \\
\hline & & & $\mathrm{R}$ & $2.87 \pm 0.01$ & $-0.30 \pm 0.01$ & $2.85 \pm 0.01$ & $48.0 \pm 0.1$ & & & \\
\hline \multirow[t]{4}{*}{$\mathrm{HD} 1337^{a}$} & free & 2015-04-30 03:47:04 & & & & & & $\ldots$ & $\cdots$ & $\cdots$ \\
\hline & & & $\mathrm{B}$ & $0.56 \pm 0.03$ & $0.04 \pm 0.02$ & $0.56 \pm 0.03$ & $42.8 \pm 1.7$ & & & \\
\hline & & & V & $0.54 \pm 0.02$ & $-0.04 \pm 0.02$ & $0.54 \pm 0.02$ & $47.1 \pm 1.3$ & & & \\
\hline & & & $\mathrm{R}$ & $0.55 \pm 0.01$ & $-0.03 \pm 0.01$ & $0.55 \pm 0.01$ & $46.6 \pm 0.7$ & & & \\
\hline HD1337 ${ }^{a}$ & free & 2015-04-30 03:55:09 & & & & & & . & $\ldots$ & $\ldots$ \\
\hline & & & $\mathrm{B}$ & $0.54 \pm 0.01$ & $-0.02 \pm 0.01$ & $0.54 \pm 0.01$ & $46.3 \pm 0.5$ & & & \\
\hline & & & V & $0.56 \pm 0.01$ & $-0.04 \pm 0.01$ & $0.56 \pm 0.01$ & $47.2 \pm 0.5$ & & & \\
\hline & & & $\mathrm{R}$ & $0.55 \pm 0.01$ & $-0.04 \pm 0.01$ & $0.55 \pm 0.01$ & $47.1 \pm 0.3$ & & & \\
\hline $\mathrm{HD} 1337^{a}$ & free & 2015-04-30 04:09:15 & & & & & & $\cdots$ & . & . \\
\hline & & & B & $0.58 \pm 0.01$ & $-0.04 \pm 0.01$ & $0.57 \pm 0.01$ & $47.0 \pm 0.6$ & & & \\
\hline & & & V & $0.58 \pm 0.01$ & $-0.05 \pm 0.01$ & $0.57 \pm 0.01$ & $47.6 \pm 0.6$ & & & \\
\hline & & & $\mathrm{R}$ & $0.56 \pm 0.01$ & $-0.04 \pm 0.01$ & $0.56 \pm 0.01$ & $46.8 \pm 0.4$ & & & \\
\hline HD137569 $9^{a, b}$ & free & 2015-04-29T23:40:10 & & & & & & $\cdots$ & $\cdots$ & $\cdots$ \\
\hline & & & $\mathrm{B}$ & $0.19 \pm 0.03$ & $-0.19 \pm 0.03$ & $-0.04 \pm 0.03$ & $\ldots$ & & & \\
\hline & & & V & $0.34 \pm 0.05$ & $-0.29 \pm 0.05$ & $-0.18 \pm 0.05$ & $\ldots$ & & & \\
\hline & & & $\mathrm{R}$ & $0.21 \pm 0.03$ & $-0.21 \pm 0.03$ & $-0.04 \pm 0.03$ & $\ldots$ & & & \\
\hline HD137569 $9^{a, b}$ & free & 2015-04-30Т00:08:05 & & & & & & $\cdots$ & $\ldots$ & $\cdots$ \\
\hline & & & B & $0.20 \pm 0.02$ & $-0.14 \pm 0.03$ & $-0.15 \pm 0.02$ & $\ldots$ & & & \\
\hline & & & V & $0.18 \pm 0.04$ & $-0.11 \pm 0.04$ & $-0.14 \pm 0.04$ & $\ldots$ & & & \\
\hline & & & $\mathrm{R}$ & $0.16 \pm 0.03$ & $-0.15 \pm 0.03$ & $-0.06 \pm 0.02$ & $\ldots$ & & & \\
\hline HD154445 & free & 2015-04-30 00:39:26 & & & & & & $5550.8 \pm 13.8$ & $3.67 \pm 0.01$ & $1.50 \pm 0.04$ \\
\hline & & & $\mathrm{B}$ & $3.37 \pm 0.01$ & $-3.36 \pm 0.01$ & $0.19 \pm 0.01$ & $88.4 \pm 0.1$ & & & \\
\hline & & & V & $3.63 \pm 0.01$ & $-3.63 \pm 0.01$ & $0.07 \pm 0.01$ & $89.4 \pm 0.1$ & & & \\
\hline & & & $\mathrm{R}$ & $3.53 \pm 0.01$ & $-3.53 \pm 0.01$ & $0.02 \pm 0.01$ & $89.9 \pm 0.1$ & & & \\
\hline HD154445 & free & 2015-04-30 04:33:46 & & & & & & $5640.8 \pm 20.5$ & $3.61 \pm 0.01$ & $1.85 \pm 0.07$ \\
\hline & & & $\mathrm{B}$ & $3.23 \pm 0.01$ & $-3.22 \pm 0.01$ & $0.13 \pm 0.01$ & $88.9 \pm 0.1$ & & & \\
\hline & & & V & $3.57 \pm 0.01$ & $-3.57 \pm 0.01$ & $0.05 \pm 0.01$ & $89.6 \pm 0.1$ & & & \\
\hline & & & $\mathrm{R}$ & $3.48 \pm 0.01$ & $-3.48 \pm 0.01$ & $-0.01 \pm 0.01$ & $90.1 \pm 0.1$ & & & \\
\hline HD194092 & free & 2015-04-30 01:21:46 & & & & & & $5727.8 \pm 235.1$ & $0.64 \pm 0.01$ & $1.46 \pm 0.47$ \\
\hline & & & $\mathrm{B}$ & $0.51 \pm 0.01$ & $-0.39 \pm 0.01$ & $0.32 \pm 0.01$ & $70.6 \pm 0.5$ & & & \\
\hline & & & V & $0.61 \pm 0.01$ & $-0.52 \pm 0.01$ & $0.31 \pm 0.02$ & $74.5 \pm 0.6$ & & & \\
\hline & & & $\mathrm{R}$ & $0.61 \pm 0.01$ & $-0.50 \pm 0.01$ & $0.34 \pm 0.01$ & $72.9 \pm 0.4$ & & & \\
\hline HD28446 & free & 2015-04-29 20:44:12 & & & & & & $4835.7 \pm 117.9$ & $2.12 \pm 0.01$ & $1.08 \pm 0.16$ \\
\hline & & & $\mathrm{B}$ & $2.13 \pm 0.02$ & $1.16 \pm 0.02$ & $-1.79 \pm 0.02$ & $151.4 \pm 0.2$ & & & \\
\hline & & & V & $2.07 \pm 0.01$ & $1.13 \pm 0.01$ & $-1.74 \pm 0.01$ & $151.6 \pm 0.2$ & & & \\
\hline & & & $\mathrm{R}$ & $1.98 \pm 0.01$ & $1.12 \pm 0.01$ & $-1.63 \pm 0.01$ & $152.3 \pm 0.1$ & & & \\
\hline HD28446 & free & 2015-04-29 20:52:17 & & & & & & $4887.0 \pm 100.4$ & $2.07 \pm 0.01$ & $0.60 \pm 0.08$ \\
\hline & & & B & $2.03 \pm 0.01$ & $1.12 \pm 0.01$ & $-1.69 \pm 0.01$ & $151.8 \pm 0.1$ & & & \\
\hline & & & V & $2.03 \pm 0.01$ & $1.10 \pm 0.01$ & $-1.70 \pm 0.01$ & $151.5 \pm 0.1$ & & & \\
\hline & & & $\mathrm{R}$ & $1.96 \pm 0.01$ & $1.09 \pm 0.01$ & $-1.63 \pm 0.01$ & $151.8 \pm 0.1$ & & & \\
\hline HD43384 & free & 2015-04-29 20:14:24 & & & & & & $5342.5 \pm 42.8$ & $2.96 \pm 0.01$ & $0.96 \pm 0.06$ \\
\hline & & & $\mathrm{B}$ & $2.88 \pm 0.01$ & $2.62 \pm 0.01$ & $-1.19 \pm 0.01$ & $167.8 \pm 0.1$ & & & \\
\hline & & & V & $2.94 \pm 0.01$ & $2.73 \pm 0.01$ & $-1.08 \pm 0.01$ & $169.2 \pm 0.1$ & & & \\
\hline & & & $\mathrm{R}$ & $2.85 \pm 0.01$ & $2.70 \pm 0.01$ & $-0.92 \pm 0.01$ & $170.6 \pm 0.1$ & & & \\
\hline HD43384 & free & 2015-04-29 21:14:04 & & & & & & $5215.5 \pm 75.8$ & $3.00 \pm 0.01$ & $0.80 \pm 0.08$ \\
\hline & & & $\mathrm{B}$ & $2.93 \pm 0.02$ & $2.72 \pm 0.02$ & $-1.08 \pm 0.03$ & $169.2 \pm 0.2$ & & & \\
\hline & & & $\mathrm{V}$ & $2.97 \pm 0.02$ & $2.79 \pm 0.01$ & $-1.02 \pm 0.02$ & $170.0 \pm 0.2$ & & & \\
\hline & & & $\mathrm{R}$ & $2.87 \pm 0.01$ & $2.72 \pm 0.01$ & $-0.91 \pm 0.01$ & $170.8 \pm 0.1$ & & & \\
\hline
\end{tabular}

Notes. The errors in this table are statistical only, while the root-mean-square of the Stokes $Q$ and $U$ is $\sim 0.04 \%$. $^{(a)}$ The constant polarization curve of this star could not be fitted well with a Serkowski curve. ${ }^{(b)}$ The polarization angle could not be determined due to low polarization degree. 
A. Cikota et al.: Spectropolarimetry of Galactic stars with anomalous extinction sightlines

Table C.3. Individual epochs with AFOSC

\begin{tabular}{|c|c|c|c|c|c|c|c|c|c|c|}
\hline \multirow[b]{2}{*}{ Name } & \multirow[b]{2}{*}{ Filter } & \multirow[b]{2}{*}{ Epoch } & \multirow[b]{2}{*}{ Passband } & \multirow[b]{2}{*}{$P(\%)$} & \multirow[b]{2}{*}{$P_{Q}(\%)$} & \multirow[b]{2}{*}{$P_{U}(\%)$} & \multirow[b]{2}{*}{$\theta\left(^{\circ}\right)$} & \multicolumn{3}{|c|}{ Serkowski curve } \\
\hline & & & & & & & & $\lambda_{\max }(\AA)$ & $P_{\max }(\%)$ & $K$ \\
\hline \multirow[t]{4}{*}{ HD 28446} & free & 2015-02-09 19:43:28 & & & & & & $4722 \pm 49$ & $2.00 \pm 0.01$ & $0.72 \pm 0.04$ \\
\hline & & & $\mathrm{B}$ & $1.99 \pm 0.01$ & $1.16 \pm 0.01$ & $-1.62 \pm 0.01$ & $152.8 \pm 0.1$ & & & \\
\hline & & & $\mathrm{V}$ & $1.96 \pm 0.01$ & $1.12 \pm 0.01$ & $-1.61 \pm 0.01$ & $152.3 \pm 0.1$ & & & \\
\hline & & & $\mathrm{R}$ & $1.85 \pm 0.01$ & $1.04 \pm 0.01$ & $-1.53 \pm 0.01$ & $152.1 \pm 0.1$ & & & \\
\hline \multirow[t]{4}{*}{ HD 43384} & free & 2015-02-09 20:03:30 & & & & & & $5201 \pm 17$ & $3.16 \pm 0.01$ & $1.03 \pm 0.02$ \\
\hline & & & $\mathrm{B}$ & $3.08 \pm 0.01$ & $2.92 \pm 0.01$ & $-0.96 \pm 0.01$ & $170.9 \pm 0.1$ & & & \\
\hline & & & $\mathrm{V}$ & $3.14 \pm 0.01$ & $3.01 \pm 0.01$ & $-0.92 \pm 0.01$ & $171.5 \pm 0.1$ & & & \\
\hline & & & $\mathrm{R}$ & $2.99 \pm 0.01$ & $2.87 \pm 0.01$ & $-0.84 \pm 0.01$ & $171.9 \pm 0.1$ & & & \\
\hline \multirow[t]{4}{*}{ HD 43384} & free & 2015-02-10 17:53:17 & & & & & & $5371 \pm 14$ & $3.19 \pm 0.01$ & $1.21 \pm 0.03$ \\
\hline & & & $\mathrm{B}$ & $3.04 \pm 0.01$ & $2.91 \pm 0.01$ & $-0.89 \pm 0.01$ & $171.5 \pm 0.1$ & & & \\
\hline & & & $\mathrm{V}$ & $3.17 \pm 0.01$ & $3.04 \pm 0.01$ & $-0.92 \pm 0.01$ & $171.6 \pm 0.1$ & & & \\
\hline & & & $\mathrm{R}$ & $3.03 \pm 0.01$ & $2.91 \pm 0.01$ & $-0.86 \pm 0.01$ & $171.8 \pm 0.1$ & & & \\
\hline \multirow[t]{4}{*}{ HD 43384} & free & 2015-03-09 21:06:00 & & & & & & $5317 \pm 20$ & $2.92 \pm 0.01$ & $1.14 \pm 0.03$ \\
\hline & & & B & $2.82 \pm 0.01$ & $2.62 \pm 0.01$ & $-1.04 \pm 0.01$ & $169.2 \pm 0.1$ & & & \\
\hline & & & V & $2.90 \pm 0.01$ & $2.71 \pm 0.01$ & $-1.02 \pm 0.01$ & $169.7 \pm 0.1$ & & & \\
\hline & & & $\mathrm{R}$ & $2.76 \pm 0.01$ & $2.60 \pm 0.01$ & $-0.93 \pm 0.01$ & $170.2 \pm 0.1$ & & & \\
\hline \multirow[t]{4}{*}{$\operatorname{HD} 1337^{a}$} & free & 2015-02-09 18:40:10 & & & & & & & & $\cdots$ \\
\hline & & & $\mathrm{B}$ & $0.51 \pm 0.01$ & $-0.12 \pm 0.01$ & $0.49 \pm 0.01$ & $52.1 \pm 0.2$ & & & \\
\hline & & & $\mathrm{V}$ & $0.50 \pm 0.01$ & $-0.14 \pm 0.01$ & $0.48 \pm 0.01$ & $53.2 \pm 0.2$ & & & \\
\hline & & & $\mathrm{R}$ & $0.54 \pm 0.01$ & $-0.19 \pm 0.01$ & $0.51 \pm 0.01$ & $55.2 \pm 0.1$ & & & \\
\hline \multirow[t]{4}{*}{$\operatorname{HD} 1337^{a}$} & free & 2015-03-10 18:06:42 & & & & & & $\ldots$ & $\ldots$ & $\ldots$ \\
\hline & & & B & $0.65 \pm 0.01$ & $-0.07 \pm 0.01$ & $0.64 \pm 0.01$ & $48.0 \pm 0.2$ & & & \\
\hline & & & $\mathrm{V}$ & $0.61 \pm 0.01$ & $-0.08 \pm 0.01$ & $0.60 \pm 0.01$ & $48.6 \pm 0.2$ & & & \\
\hline & & & $\mathrm{R}$ & $0.61 \pm 0.01$ & $-0.11 \pm 0.01$ & $0.60 \pm 0.01$ & $49.9 \pm 0.1$ & & & \\
\hline \multirow[t]{4}{*}{ HD 54439} & free & 2015-02-10 20:48:43 & & & & & & $5138 \pm 114$ & $0.69 \pm 0.01$ & $1.28 \pm 0.22$ \\
\hline & & & $\mathrm{B}$ & $0.66 \pm 0.01$ & $-0.03 \pm 0.01$ & $-0.66 \pm 0.01$ & $133.8 \pm 0.2$ & & & \\
\hline & & & V & $0.69 \pm 0.01$ & $0.08 \pm 0.01$ & $-0.68 \pm 0.01$ & $138.4 \pm 0.1$ & & & \\
\hline & & & $\mathrm{R}$ & $0.63 \pm 0.01$ & $0.03 \pm 0.01$ & $-0.63 \pm 0.01$ & $136.5 \pm 0.1$ & & & \\
\hline \multirow[t]{4}{*}{ HD 21291} & free & 2015-02-10 17:23:13 & & & & & & $5166 \pm 27$ & $2.95 \pm 0.01$ & $1.05 \pm 0.04$ \\
\hline & & & $\mathrm{B}$ & $2.87 \pm 0.01$ & $-1.73 \pm 0.01$ & $-2.29 \pm 0.01$ & $116.4 \pm 0.1$ & & & \\
\hline & & & $\mathrm{V}$ & $2.92 \pm 0.01$ & $-1.81 \pm 0.01$ & $-2.29 \pm 0.01$ & $115.9 \pm 0.1$ & & & \\
\hline & & & $\mathrm{R}$ & $2.77 \pm 0.01$ & $-1.74 \pm 0.01$ & $-2.16 \pm 0.01$ & $115.6 \pm 0.1$ & & & \\
\hline \multirow[t]{4}{*}{ HD $137569^{a, b}$} & free & 2015-02-11 03:51:49 & & & & & & $\ldots$ & $\ldots$ & $\ldots$ \\
\hline & & & B & $0.22 \pm 0.01$ & $-0.22 \pm 0.01$ & $-0.02 \pm 0.01$ & $\ldots$ & & & \\
\hline & & & $\mathrm{V}$ & $0.19 \pm 0.01$ & $-0.19 \pm 0.01$ & $-0.00 \pm 0.01$ & $\ldots$ & & & \\
\hline & & & $\mathrm{R}$ & $0.19 \pm 0.01$ & $-0.19 \pm 0.01$ & $0.01 \pm 0.01$ & $\ldots$ & & & \\
\hline \multirow[t]{4}{*}{ HD $137569^{a, b}$} & free & 2015-03-10 02:41:10 & & & & & & $\cdots$ & $\ldots$ & $\cdots$ \\
\hline & & & B & $0.20 \pm 0.01$ & $-0.18 \pm 0.01$ & $0.10 \pm 0.01$ & $\ldots$ & & & \\
\hline & & & $\mathrm{V}$ & $0.18 \pm 0.01$ & $-0.17 \pm 0.01$ & $0.07 \pm 0.01$ & $\ldots$ & & & \\
\hline & & & $\mathrm{R}$ & $0.22 \pm 0.01$ & $-0.20 \pm 0.01$ & $0.10 \pm 0.01$ & $\ldots$ & & & \\
\hline HD 198478 & free & 2016-08-02 21:00:02 & & & & & & $5132 \pm 41$ & $2.77 \pm 0.01$ & $0.99 \pm 0.06$ \\
\hline & & & $\mathrm{B}$ & $2.70 \pm 0.01$ & $2.69 \pm 0.01$ & $0.25 \pm 0.01$ & $2.6 \pm 0.1$ & & & \\
\hline & & & $\mathrm{V}$ & $2.73 \pm 0.01$ & $2.72 \pm 0.01$ & $0.30 \pm 0.01$ & $3.2 \pm 0.1$ & & & \\
\hline & & & $\mathrm{R}$ & $2.61 \pm 0.01$ & $2.59 \pm 0.01$ & $0.30 \pm 0.01$ & $3.3 \pm 0.1$ & & & \\
\hline HD 194092 & free & 2016-08-02 20:22:18 & & & & & & $5884 \pm 107$ & $0.74 \pm 0.01$ & $1.09 \pm 0.18$ \\
\hline & & & B & $0.69 \pm 0.01$ & $-0.67 \pm 0.01$ & $0.16 \pm 0.01$ & $83.1 \pm 0.3$ & & & \\
\hline & & & $\mathrm{V}$ & $0.75 \pm 0.01$ & $-0.72 \pm 0.01$ & $0.20 \pm 0.01$ & $82.4 \pm 0.2$ & & & \\
\hline & & & $\mathrm{R}$ & $0.73 \pm 0.01$ & $-0.70 \pm 0.01$ & $0.17 \pm 0.01$ & $83.1 \pm 0.1$ & & & \\
\hline
\end{tabular}

Notes. The errors in this table are statistical only, while the root-mean-square of the Stokes $Q$ and $U$ is $\sim 0.06 \%$. ${ }^{(a)}$ The constant polarization curve of this star could not be fitted well with a Serkowski curve. ${ }^{(b)}$ The polarization angle could not be determined due to low polarization degree. 